\title{
Discovery of a 310 Day Period from the Enshrouded Massive System NaSt1 (WR 122)
}

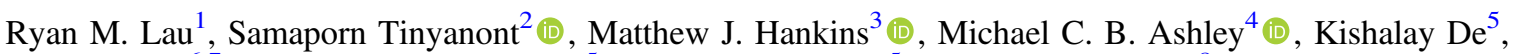 \\ Alexei V. Filippenko ${ }^{6,7}$ (D), Lynne A. Hillenbrand ${ }^{5}$, Mansi M. Kasliwal ${ }^{5}$ (D), Jon C. Mauerhan ${ }^{8}$ (D), Anthony F. J. Moffat ${ }^{9}$ (D), \\ Anna M. Moore ${ }^{10}$, Nathan Smith ${ }^{11}$ (D), Jamie Soon ${ }^{10}$, Roberto Soria ${ }^{12}$ (D), Tony Travouillon ${ }^{10}$, Karel A. van der Hucht ${ }^{13}$, \\ Peredur M. Williams ${ }^{14}$ (D), and WeiKang Zheng ${ }^{6}$ (D) \\ ${ }^{1}$ Institute of Space \& Astronautical Science, Japan Aerospace Exploration Agency, 3-1-1 Yoshinodai, Chuo-ku, Sagamihara, Kanagawa 252-5210, Japan \\ ryanlau@ir.isas.jaxa.jp \\ ${ }^{2}$ Department of Astronomy and Astrophysics, University of California, 1156 High St., Santa Cruz, CA 95064, USA \\ ${ }^{3}$ Department of Physical Sciences, Arkansas Tech University, 1701 N. Boulder Avenue, Russellville, AR 72801, USA \\ ${ }^{4}$ School of Physics, University of New South Wales, Sydney NSW 2052, Australia \\ ${ }^{5}$ Division of Physics, Mathematics, and Astronomy, California Institute of Technology, Pasadena, CA 91125, USA \\ ${ }^{6}$ Department of Astronomy, University of California, Berkeley, CA 94720-3411, USA \\ ${ }^{7}$ Miller Institute for Basic Research in Science, University of California, Berkeley, CA 94720, USA \\ ${ }^{8}$ The Aerospace Corporation, 2310 E. El Segundo Boulevard, El Segundo, CA 90245 , USA \\ ${ }^{9}$ Département de Physique, Université de Montréal, C.P. 6128, succ. centre-ville, Montréal (Qc) H3C 3J7, Canada, and Centre de Recherche en Astrophysique du \\ Québec, Canada \\ ${ }^{10}$ Research School of Astronomy and Astrophysics, Australian National University, Canberra, ACT 2611, Australia \\ ${ }^{11}$ University of Arizona, Steward Observatory, 933 N. Cherry Avenue, Tucson, AZ 85721, USA \\ ${ }^{12}$ National Astronomical Observatories, Chinese Academy of Sciences, Beijing 100012, People's Republic of China \\ ${ }^{13}$ SRON Netherlands Institute for Space Research Sorbonnelaan 2, NL-3584CA Utrecht, The Netherlands \\ ${ }^{14}$ Institute for Astronomy, University of Edinburgh, Royal Observatory, Edinburgh EH9 3HJ, UK \\ Received 2021 March 15; revised 2021 August 27; accepted 2021 August 27; published 2021 November 15
}

\begin{abstract}
We present optical and infrared (IR) light curves of NaSt1, also known as Wolf-Rayet 122, with observations from Palomar Gattini-IR (PGIR), the Zwicky Transient Facility (ZTF), the Katzman Automatic Imaging Telescope, the Asteroid Terrestrial-impact Last Alert System, and the All-Sky Automated Survey for Supernovae (ASAS-SN). We identify a $P=309.7 \pm 0.7$ day photometric period from the optical and IR light curves that reveal periodic, sinusoidal variability between 2014 July and 2021 July. We also present historical IR light curves taken between 1983 July and 1989 May, which show variability consistent with the period of the present-day light curves. In the past, NaSt1 was brighter in the $J$ band with larger variability amplitudes than the present-day PGIR values, suggesting that NaSt1 exhibits variability on longer ( $\gtrsim$ decade) timescales. Sinusoidal fits to the recent optical and IR light curves show that the amplitude of NaSt1's variability differs at various wavelengths and also reveal significant phase offsets of $17.0 \pm 2.5$ day between the ZTF $r$ and PGIR $J$ light curves. We interpret the 310 day photometric period from NaSt1 as the orbital period of an enshrouded massive binary. We suggest that the photometric variability of NaSt1 may arise from variations in the line-of-sight optical depth toward circumstellar optical/IR-emitting regions throughout its orbit due to colliding-wind dust formation. We speculate that past mass transfer in NaSt1 may have been triggered by Roche-lobe overflow (RLOF) during an eruptive phase of an Ofpe/WN9 star. Lastly, we argue that NaSt1 is no longer undergoing RLOF mass transfer.
\end{abstract}

Unified Astronomy Thesaurus concepts: Massive stars (732); Interacting binary stars (801); Wolf-Rayet stars (1806); Light curves (918); Circumstellar dust (236)

Supporting material: machine-readable table

\section{Introduction}

Almost all massive stars have a close binary companion ( $P \lesssim 5000$ day), implying that binary interaction is common in massive stars (Sana et al. 2012; Moe \& Di Stefano 2017). The ubiquity of binary interactions such as mass transfer has challenged our understanding of massive-star evolution and notably diverges from the theoretical framework of single-star evolution (e.g., Heger et al. 2003; Groh et al. 2013). Binary interaction can influence mass loss and enable additional massive-star evolutionary pathways beyond the single-star evolution framework (Smith 2014). In particular, Roche-lobe overflow (RLOF) is a critical binary mass-transfer processes that can strip the hydrogen envelope of a massive star and lead to the emergence of a helium-rich Wolf-Rayet (WR) star (Paczyński 1967; Podsiadlowski et al. 1992; De Marco \& Izzard 2017; Götberg et al. 2018). Agreement between the observed statistical properties of massive stars and predictions from recent binary stellar-population synthesis models indeed indicate that binary interactions are important in the formation of WR stars (Vanbeveren et al. 1998; Meynet \& Maeder 2005; Rosslowe \& Crowther 2015; Eldridge et al. 2017).

The WR phase is an important evolved stage of a massive star that precedes its end-of-life explosion as a Type Ib/c supernova $(\mathrm{SN})$ or direct collapse to a black hole (Heger et al. 2003; Smith et al. 2011a; Sukhbold et al. 2016). WR binaries may therefore precede the formation of binary black holes and are potential progenitors of black-hole mergers (e.g., Belczynski et al. 2016). Populations of WR stars formed via binary interaction can also have a profound impact on the ionization of the interstellar medium (ISM) of their host galaxies as well as the intergalactic medium beyond (Götberg et al. 2020; Sander \& Vink 2020). WR star-dust formation, which has been observed in colliding winds from carbon-rich 
WR (WC) stars with OB-star binary companions (e.g., Williams et al. 1987; Tuthill et al. 1999), may also significantly contribute to dust abundances in the ISM of their host galaxies (Lau et al. 2020).

Understanding the underlying physics of binary interactions that lead to the formation of a WR star is therefore of great importance. However, there is a dearth of known interacting massive binaries owing to the relatively short timescales associated with the RLOF mass-transfer process in post-mainsequence (Case B) mass transfer. One of the most well-known interacting massive binary systems that is currently undergoing RLOF mass transfer is RY Scuti, which is an eclipsing system with an $\sim 11$ day orbital period surrounded by a toroidal circumstellar nebula of dust and ionized gas (Gehrz et al. 1995; Smith et al. 1999, 2002; Grundstrom et al. 2007; Smith et al. 2011b).

The enigmatic NaSt1 system (also known as LS IV +005 and WR 122) was initially classified as a late-type, nitrogenrich WR (WN10) star by Nassau \& Stephenson (1963) and Massey \& Conti (1983) but was later proposed to be a B[e], O [e], or Ofpe/WN9 star (van der Hucht et al. 1989, 1997). NaStl again had its true nature called into question as a result of high spectral resolution observations by Crowther \& Smith (1999), who suggested that NaSt1 may be an early-type WR star enshrouded by an $\eta$ Car-like nebula. It is now thought to host a WR star emerging from an RLOF mass-transfer phase with a binary companion (Mauerhan et al. 2015). NaSt1 is an intriguing system that may present a rare opportunity to investigate this transitional phase, particularly given its relatively nearby distance of $3.03_{-0.45}^{+0.60} \mathrm{kpc}$ (Rate \& Crowther 2020).

The presence of a binary system in NaSt1 was inferred from its surrounding extended $\left(\sim 7^{\prime \prime}\right)$ disk-like, nitrogen-rich nebula which is thought to originate from nonconservative mass transfer in the central system (Mauerhan et al. 2015). Mauerhan et al. (2015) also showed that NaStl exhibits X-ray emission consistent with colliding-wind WR binaries. The orbital and stellar properties of the central binary are important diagnostics of mass transfer via RLOF; however, these properties remain largely unknown for $\mathrm{NaSt} 1$.

NaSt 1 possesses an interesting combination of observational characteristics that are important for understanding the nature of its stellar component(s). Optical and near-infrared (IR) spectroscopy by Crowther \& Smith (1999) showed no obvious stellar lines but revealed narrow nebular emission features consistent with fully CNO-processed material that is thought to be ionized by an unseen WR star. The unusual chemical composition of NaSt1's nebula has notably drawn comparisons to the luminous blue variable (LBV) system $\eta$ Car and its surrounding N-rich nebula (Smith \& Morse 2004). Highexcitation emission lines from NaSt 1 also indicated that the ionizing source of its nebula must possess an effective temperature $\gtrsim 30,000 \mathrm{~K}$, which is consistent with the presence of a WR star. Classical WR stars, however, are characterized by broad, full-width at half-maximum intensity $\gtrsim 1000 \mathrm{~km} \mathrm{~s}^{-1}$ emission features. These broad features are notably absent from NaSt1's spectrum. Interestingly, many of the strong emission lines from NaSt1 exhibit double-peaked profiles separated by tens of $\mathrm{km} \mathrm{s}^{-1}$, which may originate from a circumstellar or circumbinary disk that could be obscuring or outshining WR emission features (Mauerhan et al. 2015). Similar multi-peaked narrow emission features dominate the optical spectrum in RY
Scuti (Smith et al. 2002). NaSt1's nebular spectrum is also heavily reddened $\left(A_{V} \approx 6.5 \mathrm{mag}\right.$; Crowther \& Smith 1999) by interstellar and/or circumstellar material. Strong thermal-IR emission from NaSt1 indicates the presence of circumstellar dust that either formed recently or is currently forming (Rajagopal et al. 2007).

In this paper, we present the discovery of periodic photometric variability from $\mathrm{NaSt} 1$ with data from optical and near-IR imaging surveys. This work also presents some of the first science results from the recently commissioned near-IR imaging survey Palomar Gattini-IR (PGIR; Moore \& Kasliwal 2019; De et al. 2020a). In Section 2, we describe the new and archival observations of NaSt1 used in our analysis. The results of our optical and IR variability analysis of $\mathrm{NaSt} 1$ are presented in Section 3. In Section 4, we discuss the possible origins for the observed photometric variability and speculate on the nature of $\mathrm{NaSt} 1$, including whether it is currently undergoing mass transfer. We summarize our results and conclude in Section 5.

\section{Observations and Archival Data}

\subsection{PGIR J-band Photometry}

PGIR is a wide-field, time-domain imaging survey located at Palomar Observatory. It utilizes a $30 \mathrm{~cm}$ telescope and $J$-band filter similar to the 2MASS $J$ filter with an effective wavelength of $\lambda_{\text {eff }}=1.235 \mu \mathrm{m}$ and an effective filter bandwidth of $\Delta \lambda=0.1624 \mu \mathrm{m}$. PGIR achieves a $4.96 \times 4.96$ field of view $(\mathrm{FoV})$ with its $2 \mathrm{k} \times 2 \mathrm{k}$ HAWAII-2RG detector and a scale of 8 ". 7 pixel $^{-1}$. The entire sky visible from Palomar is imaged by PGIR every $\sim 2$ nights down to a $5 \sigma$ detection limit of 14.8 Vega mag. ${ }^{15}$ The PGIR image processing and photometric calibration are described by De et al. (2020a). The $J$-band saturation limit of PGIR was initially $\sim 8.5 \mathrm{mag}$, but has been subsequently improved to $J \approx 6$ mag after implementing a new readout mode described by De et al. (2020b).

NaSt 1 , which is located at $\alpha(\mathrm{J} 2000)=18^{\mathrm{h}} 52^{\mathrm{m}} 17.55$ and $\delta$ $(\mathrm{J} 2000)=+00^{\circ} 59^{\prime} 44^{\prime \prime} 3$ (Gaia Collaboration 2018), was well covered by PGIR imaging fields. In order to investigate the $J$-band variability of NaSt1, forced-aperture photometry was performed at its coordinates using a 3 resampled-pixel radius $\left(\sim 13^{\prime \prime}\right)$ aperture in PGIR images taken between 2018 November and 2021 June. Importantly, source confusion is not an issue since NaSt1 is $>3.8$ mag brighter in $J$ than all objects within $15^{\prime \prime}$ of its coordinates (Cutri et al. 2003). Photometry from PGIR and all other platforms included in this work are provided in Table 1 .

\subsection{KAIT Optical Photometry}

Optical BVRI photometry of NaSt1 was obtained with the robotic $0.76 \mathrm{~m}$ Katzman Automatic Imaging Telescope (KAIT; Filippenko et al. 2001) at Lick Observatory. Additional Clearband (close to the $R$ band; see Li et al. 2003) images were also obtained with KAIT. A series of 66 imaging observations of NaSt1 was performed with KAIT between 2014 July and 2015 November. The KAIT observing frequency of NaSt1 ranged from a few days to a few weeks when visible.

All images were reduced using a custom pipeline (Ganeshalingam et al. 2010; Stahl et al. 2019). Point-spread-function (PSF) photometry was then obtained using DAOPHOT

\footnotetext{
15 All magnitudes provided in this work are in the Vega magnitude system.
} 
Table 1

KAIT, ATLAS, ASAS-SN, ZTF, and PGIR Photometry of NaSt1

\begin{tabular}{|c|c|c|c|c|c|c|c|c|c|c|}
\hline MJD & $\begin{array}{c}B \\
\text { (KAIT) }\end{array}$ & $\begin{array}{c}g \\
(\mathrm{ZTF})\end{array}$ & $\begin{array}{c}c \\
(\text { ATLAS) }\end{array}$ & $\begin{array}{c}V \\
\text { (KAIT) }\end{array}$ & $\begin{array}{c}V \\
(\mathrm{ASAS}-\mathrm{SN})\end{array}$ & $\begin{array}{c}r \\
(\mathrm{ZTF})\end{array}$ & $\begin{array}{c}R \\
\text { (KAIT) }\end{array}$ & $\begin{array}{c}o \\
(\text { ATLAS) }\end{array}$ & $\begin{array}{c}I \\
\text { (KAIT) }\end{array}$ & $\begin{array}{c}J \\
\text { (PGIR) }\end{array}$ \\
\hline $56,818.446$ & $16.21 \pm 0.04$ & - \pm- & - \pm- & $14.38 \pm 0.02$ & - \pm- & - \pm- & $12.82 \pm 0.03$ & - \pm- & $12.29 \pm 0.03$ & $\overline{- \pm-}$ \\
\hline $56,821.406$ & $16.23 \pm 0.06$ & - \pm- & - \pm- & $14.39 \pm 0.02$ & - \pm- & - \pm- & $12.82 \pm 0.03$ & - \pm- & $12.29 \pm 0.03$ & - \pm- \\
\hline $56,824.434$ & $16.22 \pm 0.07$ & - \pm- & - \pm- & $14.38 \pm 0.02$ & - \pm- & - \pm- & $12.81 \pm 0.03$ & - \pm- & $12.30 \pm 0.03$ & - \pm- \\
\hline $56,826.437$ & $16.18 \pm 0.04$ & - \pm- & - \pm- & $14.38 \pm 0.02$ & - \pm- & - \pm- & $12.80 \pm 0.03$ & - \pm- & $12.27 \pm 0.03$ & - \pm- \\
\hline$\cdots$ & $\ldots$ & $\ldots$ & $\ldots$ & $\ldots$ & $\ldots$ & $\ldots$ & $\ldots$ & $\ldots$ & $\ldots$ & $\ldots$ \\
\hline ... & $\cdots$ & $\ldots$ & $\ldots$ & $\ldots$ & $\ldots$ & $\ldots$ & $\ldots$ & $\ldots$ & $\ldots$ & $\ldots$ \\
\hline
\end{tabular}

Note. Optical/IR photometry from KAIT, ATLAS, ASAS-SN, ZTF, and PGIR. All magnitudes are given in the Vega system, and the errors correspond to the $1 \sigma$ uncertainty in the photometric measurements. A full version of this table (with the KAIT Clear photometry) is available electronically.

(This table is available in its entirety in machine-readable form.)

(Stetson 1987) from the IDL Astronomy Users Library. ${ }^{16}$ Several nearby stars were chosen from the Pan-STARRS $1^{17}$ catalog for calibration. Their magnitudes were first transformed into Landolt magnitudes (Landolt 1992) using the empirical prescription presented by Tonry et al. (2012) and then transformed to the KAIT natural system.

Apparent magnitudes were all measured in the KAIT4 natural system. The final results were transformed to the standard system using local calibrators and color terms for KAIT4 as given in Table 4 of Ganeshalingam et al. (2010), except for KAIT Clear-band data, where no reliable color term is measured owing to the broad response function. We therefore present the Clear magnitude relative to the reference stars in Landolt $R$ magnitude, which is essentially in the KAIT natural system.

\subsection{BASS Mid-IR Spectroscopy}

Mid-IR observations of NaSt1 were made using The Broadband Array Spectrograph System (BASS) sensor (Hackwell et al. 1990) at the $3.6 \mathrm{~m}$ Advanced Electro-Optical System (AEOS) telescope on Haleakela, HI, on 2017 October 23, 2018 May 13, 2019 April 6, and 2019 August 24 (UT dates are used throughout this paper). AEOS utilizes a chopping secondary mirror, run at $7 \mathrm{~Hz}$ for rapid sampling of the background. While observing, a chop-and-nod strategy was used with a chop throw of $\sim 11^{\prime \prime}$ and a telescope nod of the same distance between exposures. Integrations of $30 \mathrm{~s}$ were obtained per nod position, and 12-26 samples were obtained for each of the AEOS observations (6-13 min total integration).

An off-axis visible-wavelength CCD was used for guiding to keep the source centered in the 4." 2 instrument aperture during exposures. The resolving power of BASS is 30-125 across a 3-13 $\mu \mathrm{m}$ bandpass. Flux calibration was obtained via observations of the standard star $\alpha$ Lyr (Vega) at an airmass similar to that of the NaSt1 observations. Flux calibration was performed by dividing the target spectrum by the Vega spectrum and then multiplying the result by a Kurucz model atmosphere of Vega matched to the resolution of the data. This process also removes most of the telluric features. However, residual telluric artifacts remained, particularly in the deep water absorption band over 5-8 $\mu \mathrm{m}$, so those data were removed for clarity. Although the stellar-atmosphere model does not account for dust excess, Vega's debris excess does not emit significantly shortward of $13 \mu \mathrm{m}$. For example, at the long-wavelength end

\footnotetext{
16 http://idlastro.gsfc.nasa.gov/

17 http://archive.stsci.edu/panstarrs/search.php
}

Table 2

NaSt1 Historical IR Magnitudes

\begin{tabular}{lcccccc}
\hline \hline MJD & $J$ & $H$ & $K$ & $L^{\prime}$ & $M$ & Source \\
\hline 45,528 & 9.54 & 8.51 & 6.50 & 4.12 & & UKIRT $^{\mathrm{a}}$ \\
45,838 & 9.52 & 8.46 & 6.39 & 3.99 & 3.30 & ESO $^{\mathrm{a}}$ \\
46,237 & 9.19 & 8.27 & 6.25 & 3.90 & 3.46 & UKIRT $^{-19}$ \\
46,302 & 9.05 & 7.95 & 5.94 & 3.78 & 3.16 & UKIRT $^{-16}$ \\
46,715 & 9.49 & 8.18 & 6.19 & 3.91 & 3.18 & UKIRT \\
46,960 & 9.10 & 7.82 & 5.88 & 3.71 & 3.04 & ESO \\
47,258 & 8.99 & 7.85 & 5.87 & 3.66 & 2.98 & ESO \\
47,259 & 8.99 & 7.82 & 5.87 & 3.66 & 3.02 & ESO \\
47,261 & 9.04 & 7.89 & 5.87 & 3.68 & 2.96 & ESO \\
47,304 & 9.18 & 7.97 & 6.02 & 3.76 & 3.12 & ESO \\
47,670 & 9.40 & 8.25 & 6.25 & 3.89 & 3.19 & ESO \\
47,671 & 9.39 & 8.25 & 6.25 & 3.90 & 3.23 & ESO \\
\hline
\end{tabular}

Notes. IR photometry of NaSt1 from UKIRT and the ESO $1 \mathrm{~m}$ photometric telescope. Uncertainties are assumed to be $0.1 \mathrm{mag}$ for $J H K L^{\prime}$-band photometry and 0.15 mag for $M$-band photometry.

${ }^{a}$ Previously published by Williams et al. (1987).

of our BASS spectrum, Vega's $13 \mu \mathrm{m}$ debris disk excess is $<2 \%-3 \%$ of the photosphere ( $\mathrm{Su}$ et al. 2013).

Wavelength calibration of each BASS channel was performed in the lab using a monochrometer and either checked on-sky with observations of a strong mid-IR emission-line source (planetary nebula NGC 7027) or in the dome using a blackbody source (hot plate) measured at two temperatures $\left(40^{\circ} \mathrm{C}\right.$ and $60^{\circ} \mathrm{C}$ ) and attenuated by films of polystyrene and polysulfone to superimpose absorption features for reference.

\subsection{Historical UKIRT and ESO IR Photometry}

We present historical IR $J H K L^{\prime} M$ photometry of NaSt1 (Table 2) taken from the $3.8 \mathrm{~m}$ United Kingdom Infrared Telescope (UKIRT) and the European Southern Observatory (ESO) $1 \mathrm{~m}$ photometric telescope between 1983 July and 1989 May (MJD 45,528-47,671). The UKIRT and ESO photometry taken on 1983 July 13 (MJD 45,838) and 1983 May 18 (MJD $45,528)$, respectively, were previously published by Williams et al. (1987). Subsequent photometry is presented in this work for the first time. Since these observations were taken as a continuation of the photometry published by Williams et al. (1987), the observations follow the same procedures described in their paper. ESO $J H K L^{\prime} M$ filter properties are described by van der Bliek et al. (1996). The UKIRT $J H K$ and $L^{\prime} M$ filter properties are provided by Hawarden et al. (2001) and Leggett et al. (2003), respectively. 
UKIRT $J H K L^{\prime}$ photometry was obtained using a 12 ". 4 diameter aperture, and $M$ photometry was obtained with a $5^{\prime \prime}$ diameter aperture. ESO photometry was measured through a $15^{\prime \prime}$ diameter aperture except for the observation taken on 1987 June 14 (MJD 46,960 ), where a $22^{\prime \prime}$ diameter aperture was used. The photometry is shown in Table 2 and provided in Vega magnitudes.

\subsection{Archival Optical Photometry: ZTF, ASAS-SN, and ATLAS}

We utilize the $g$-band and $r$-band PSF-fit photometry of NaSt1 from the Zwicky Transient Facility (ZTF; Bellm et al. 2019; Masci et al. 2019) Public Data Release $6 .^{18}$ ZTF observations of NaSt1 were taken between 2018 April and 2021 April with a $\sim 1$ day cadence when visible. Only the photometry with clean extractions (i.e., "catflags $=0$ ") from ZTF DR6 were used for the analysis. Several nights included multiple observations of the field containing NaSt1. For example, high-cadence $r$-band observations were taken on MJD 58,347 and MJD 58,348, where each night consisted of $\sim 100$ images. Multiple ZTF $g$ - and $r$-band observations taken over a night were therefore averaged in single-night bins. Since ZTF photometry from the DR6 catalog was provided in units of $\mathrm{AB}$ magnitude, we converted the photometry to Vega units by applying the conversions $m_{g, \mathrm{AB}}-m_{g, \mathrm{Vega}}=-0.08$ and $m_{r, \mathrm{AB}}-$ $m_{r, \mathrm{Vega}}=0.16$.

We also utilize the $V$-band photometry of NaSt 1 from the AllSky Automated Survey for Supernovae (ASAS-SN; Shappee et al. 2014; Kochanek et al. 2017). Photometry of NaSt1 was obtained from the ASAS-SN Variable Star Database ${ }^{19}$ (Jayasinghe et al. 2020), where it was identified as a variable with the reference ID ASASSN-V J185217.55+005944.3. ASAS-SN photometry of NaSt 1 from the Variable Star Database spans from 2015 February to 2018 November with an observing cadence of $\sim 1$ week when visible.

Lastly, we use photometric measurements of NaSt1 obtained by the Asteroid Terrestrial-impact Last Alert System (ATLAS) project between 2015 October 23 (MJD 57,318) and 2021 July 9 (MJD 59,404) from the ATLAS forced-photometry server ${ }^{20}$ (Heinze et al. 2018; Tonry et al. 2018; Smith et al. 2020). ATLAS surveys the entire visible sky at declinations north of $-50^{\circ}$ every two nights with the ATLAS orange (" $o$-band"; $\lambda_{\text {eff }}=6632 \AA$ ) and cyan ("c-band"; $\lambda_{\text {eff }}=5184 \AA$ ) filters. ATLAS photometry obtained from the forced-photometry server were filtered by ignoring observations where the NaStl was within 100 pixels of the edge of the FoV and where the magnitude error was greater than $0.1 \mathrm{mag}$. Multiple observations of NaSt1 taken over a night were averaged in single-night bins. PSF photometry of NaSt1 was converted from Jy to Vega magnitudes using zero-points of 3585.79 and $2846.76 \mathrm{Jy}$ for the $c$ - and $o$-band filters, respectively, which were derived by the Spanish Virtual Observatory Filter Profile Service (Rodrigo et al. 2012; Rodrigo \& Solano 2020).

\section{Results and Analysis}

\subsection{Light-curve Periodicity, Variability Amplitude, and Phase Offset}

The near-IR and optical light curves of NaSt1 taken between 2014 July and 2021 July are shown in Figure 1 and present

\footnotetext{
18 https://www.ztf.caltech.edu/page/dr6

19 https://asas-sn.osu.edu/variables

${ }^{20}$ https://fallingstar-data.com/forcedphot/
}

clear evidence of smooth variability on a roughly year-long timescale. Since the variations are sufficiently smooth and symmetric about maxima, sinusoidal functions were fit to the PGIR, ZTF, ATLAS, ASAS-SN, and KAIT photometry using a least-squares fitting routine from the SciPy library in Python v.3.8.5. The free parameters were the period, variability amplitude, magnitude offset, and phase. The sinusoidal fits to each light curve reveal variability consistent with a period of $\sim 300$ days. The best-constrained period was derived from the fit to the ZTF $r$-band light curve of $P_{r}=309.7 \pm 0.7$ days, which we adopt as NaSt1's photometric variability period. All other light curves were then refitted with an adopted period of $P=309.7$ days. The results from the adopted-period sinusoidal fits are shown in Table 3. The following analysis utilizes the results from these adopted-period ( $P=309.7$ day) sinusoidal fits.

Interestingly, the sinusoidal variability amplitudes differ across the different wavelengths, which is shown in Figure 2. The ZTF $r$, KAIT $R$, ATLAS $o$, and PGIR $J$ bands exhibit higher-variability amplitudes relative to the other photometric bands. The KAIT Iband amplitude is comparable to the ZTF $g$ - and ATLAS $c$-band amplitudes, which demonstrates that there is no obvious trend in the amplitudes as a function of wavelength. The apparent wavelength dependence of NaSt1's variability amplitude notably differs from the expected trend for stellar pulsations (e.g., Cepheids), where an increased amplitude is expected toward shorter wavelengths (Klagyivik \& Szabados 2009).

The amplitude fit from the ASAS-SN $V$-band photometry is discrepant with the KAIT $V$-band amplitude despite the similar wavelength coverage of the instrument filters (Table 3). Owing to the much larger $8^{\prime \prime}$ pixels of ASAS-SN compared to the 0." 8 KAIT pixels and the two-pixel $\left(16^{\prime \prime}\right)$ radius aperture used for ASAS-SN photometry (Kochanek et al. 2017), the lower ASAS-SN amplitude is likely due to confusion with a nearby source of $V$-band brightness comparable to that of NaSt1. Photometry from the Pan-STARRS Data Release 1 catalog (Chambers et al. 2016) supports this explanation and shows that there is a source located $\sim 16^{\prime \prime}$ from NaSt1 with a PS1 $g$-band brightness 57\% that of NaSt1. Synthetic Johnson $V$-band photometry derived from optical spectroscopy of NaSt1 presented by Crowther \& Smith (1999), where $V=$ 14.47 mag also shows closer agreement with the 14.41 mag offset derived from the KAIT $V$-band light-curve fit (Table 3 ). The $0.066 \pm 0.004$ mag amplitude derived from the KAIT $V$-band photometry therefore more accurately traces the NaSt 1 variability than the lower-amplitude ASAS-SN photometry.

The phase-folded PGIR J-band and ZTF $r$-band light curves, which show the highest variability amplitude, are shown in Figure 3 (left). These phase-folded light curves and the fittedmodel residuals demonstrate close agreement with sinusoidal variability. The phase-folded light curves also show the cycleto-cycle consistency of NaSt1's photometric variability.

The ZTF $r$-band and PGIR $J$-band light curves from NaSt1 between MJD 58,550 and MJD 58,900 and the adopted-period sinusoidal fits indicate a significant $(>5 \sigma)$ phase offset of $+17 \pm 2.5 \mathrm{~d}$, where the $J$-band peak lags behind the $r$-band peak (Figure 3, right). There is a similar phase offset between the ZTF $r$-band and $g$-band light-curve fits, where the $g$-band offset agrees with the PGIR $J$-band offset within the uncertainties. The phase offsets suggest that the PGIR $J$-band and ZTF $r$-band emission originate from distinct stellar and/or circumstellar components of NaSt1. 


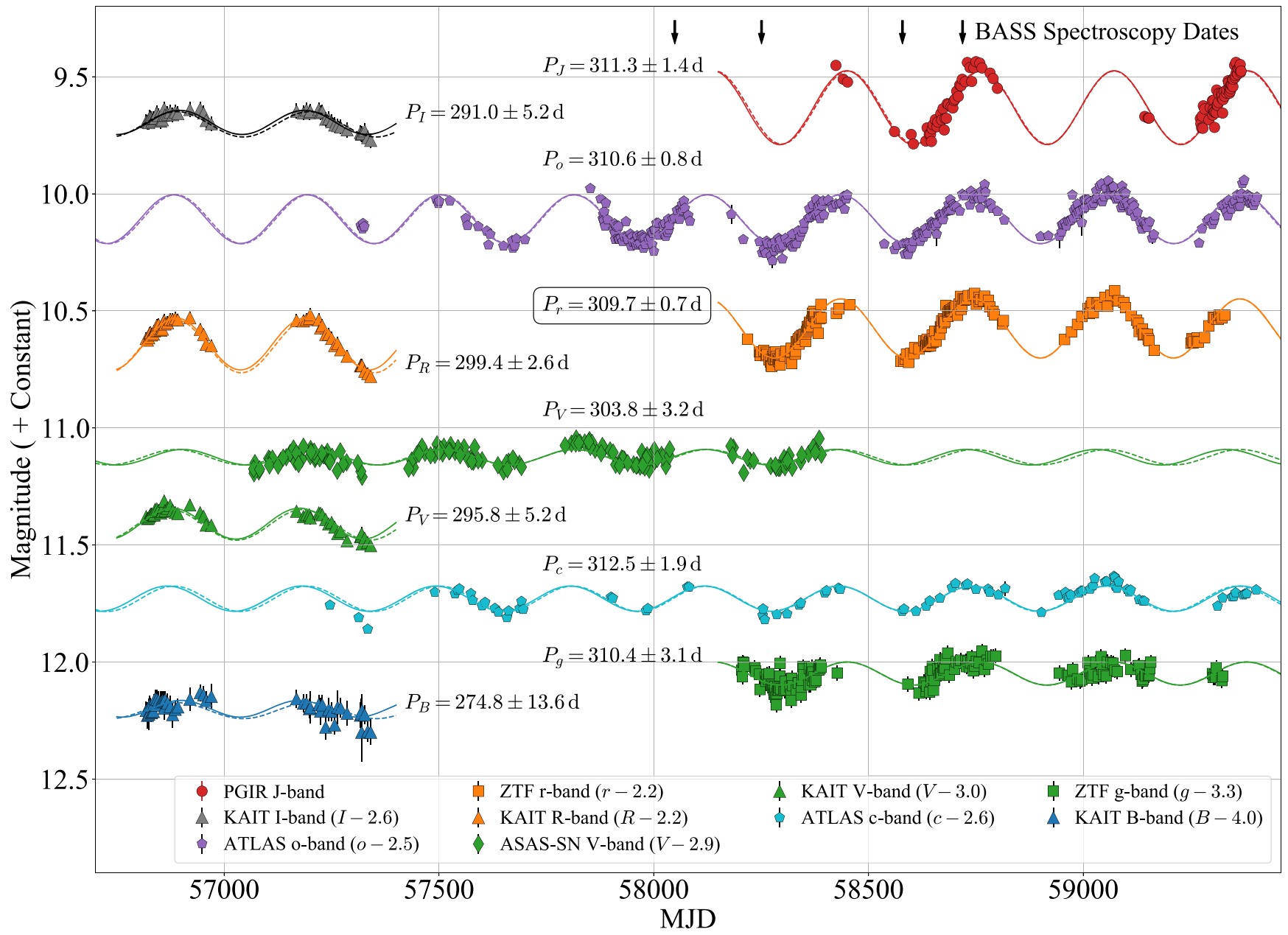

Figure 1. Optical and near-IR light curves of NaSt1 from KAIT (BVRI), ATLAS (co), ASAS-SN (V), ZTF ( $g r)$, and PGIR ( $J$ ) taken between 2014 July and 2021 July. Constant offsets have been applied to all light curves except that of PGIR, and the offset value is indicated in the legend. The light-curve plot is overlaid with individual-filter (solid line) and adopted-period (dashed line) sinusoidal fits, where the adopted-period fits assume a 309.7 day period derived from the ZTF $r$-band fit (shown in box). The similarity of the adopted-period and individual-filter fits of all light curves demonstrates agreement with a 309.7 day period. The periods derived from individual-filter fits are overlaid on the plot next to their respective light curve. Dates of the four mid-IR spectroscopic observations with BASS are also indicated on the plot as black arrows. Error bars in the light curve correspond to $1 \sigma$ uncertainties in the measured magnitudes.

Table 3

NaSt1 Adopted-Period Fit Results $(P=309.7$ day $)$

\begin{tabular}{|c|c|c|c|c|c|c|}
\hline Telescope (Filter) & Amplitude (mag) & Mag. Offset & $\operatorname{MJD}(\varphi=0)$ & $\Delta \varphi\left(r_{\mathrm{ZTF}}\right)(\mathrm{d})$ & $F_{\text {line }} / F_{\text {phot }}$ & Brightest Lines \\
\hline PGIR $(J) 12350 \AA(1624 \AA)$ & $0.156 \pm 0.006$ & $9.632 \pm 0.006$ & $58,684.8 \pm 2.3$ & $17.0 \pm 2.5$ & $<0.01$ & He I $\lambda 12785 \mathrm{He}$ I $\lambda 12526 \mathrm{~Pa} \beta$ \\
\hline KAIT $(I) 8061 \AA(1471 \AA)$ & $0.054 \pm 0.004$ & $12.303 \pm 0.002$ & $56,812.0 \pm 2.7$ & $2.3 \pm 5.2$ & $0.05 \pm 0.01$ & He II $\lambda 8237$ O I $\lambda 8446$ [N I] $\lambda 8680$ \\
\hline ATLAS $(o) 6632 \AA ⿻$ (2368 $)$ & $0.105 \pm 0.002$ & $12.608 \pm 0.002$ & $58,667.8 \pm 1.3$ & $-0.1 \pm 1.5$ & $0.18 \pm 0.03$ & He I $\lambda 6678$ He I $\lambda 5876$ He I $\lambda 7281$ \\
\hline $\mathrm{ZTF}(r) 6340 \AA(1515 \AA)$ & $0.126 \pm 0.002$ & $12.775 \pm 0.002$ & $58,667.9 \pm 0.9$ & $0.0 \pm 0.9$ & $0.23 \pm 0.03$ & He I $\lambda 6678 \mathrm{He}$ I $\lambda 5876 \mathrm{H} \alpha$ \\
\hline KAIT $(R) 6273 \AA$ (1202 ̊) & $0.114 \pm 0.004$ & $12.850 \pm 0.002$ & $56,807.5 \pm 1.3$ & $-2.1 \pm 4.6$ & $0.30 \pm 0.04$ & He I $\lambda 6678 \mathrm{He} \mathrm{I} \lambda 5876 \mathrm{H} \alpha$ \\
\hline 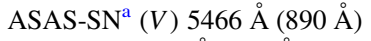 & $0.033 \pm 0.003$ & $14.025 \pm 0.002$ & $57,120.1 \pm 4.6$ & $0.8 \pm 5.9$ & $0.24 \pm 0.03$ & He I $\lambda 5876$ [N II] $\lambda 5755$ He II $\lambda 5412$ \\
\hline KAIT $(V) 5389 \AA$ (909 ̊) & $0.066 \pm 0.004$ & $14.414 \pm 0.003$ & $56,796.2 \pm 3.2$ & $-13.5 \pm 5.5$ & $0.12 \pm 0.02$ & [N II] $\lambda 5755 \mathrm{He}$ I $\lambda 5016 \mathrm{He}$ II $\lambda 5412$ \\
\hline ATLAS $(c) 5184 \AA(2144 \AA)$ & $0.054 \pm 0.004$ & $14.370 \pm 0.003$ & $58,663.5 \pm 3.5$ & $-4.4 \pm 3.6$ & $0.17 \pm 0.02$ & He I $\lambda 5876$ [N II] $\lambda 5755 \mathrm{He}$ II $\lambda 4686$ \\
\hline ZTF $(g) 4722 \AA ̊(1282 \AA)$ & $0.049 \pm 0.004$ & $15.349 \pm 0.003$ & $58,682.6 \pm 3.7$ & $14.7 \pm 3.8$ & $0.15 \pm 0.02$ & He II $\lambda 4686 \mathrm{He}$ I $\lambda 5016 \mathrm{H} \beta$ \\
\hline KAIT $(B) 4445 \AA$ ㅇ (907 ̊̊) & $0.034 \pm 0.009$ & $16.208 \pm 0.006$ & $56,811.9 \pm 9.4$ & $2.2 \pm 10.4$ & $0.24 \pm 0.03$ & He II $\lambda 4686 \mathrm{H} \beta[\mathrm{Fe}$ III] $\lambda 4658$ \\
\hline
\end{tabular}

Notes. NaSt1 light curve and adopted-period fit results, which were derived assuming a period matching the ZTF $r$-band value of $P=309.7$ day (see Figure 1 ). The listed properties include the telescope/instrument details (filter effective wavelength and bandwidth), the amplitude of the sinusoidal variability, the magnitude offset at the mid-line of the sine function, the MJD where the phase of the sine function $\varphi=0$, and the phase offset $(\Delta \varphi)$ from the PGIR value. The phase fitting for the ZTF, ATLAS, and PGIR models assumed an initial $\varphi=0$ estimate of MJD 58,700, while the KAIT and ASAS-SN models assumed initial $\varphi=0$ estimates of MJD 57,100 and MJD 56,800, respectively. The relative flux contributions from the cataloged nebular emission lines from NaSt1 (Crowther \& Smith 1999) to the photometric measurements in the different filters' wave bands (based on the adopted-period fits) are shown under $F_{\text {line }} / F_{\text {phot }}$. The brightest three lines in each filter band are also listed in descending order of line strength.

a ASAS-SN photometry of NaSt1 is likely confused with a nearby $\left(\sim 16^{\prime \prime}\right)$ optical point source. 


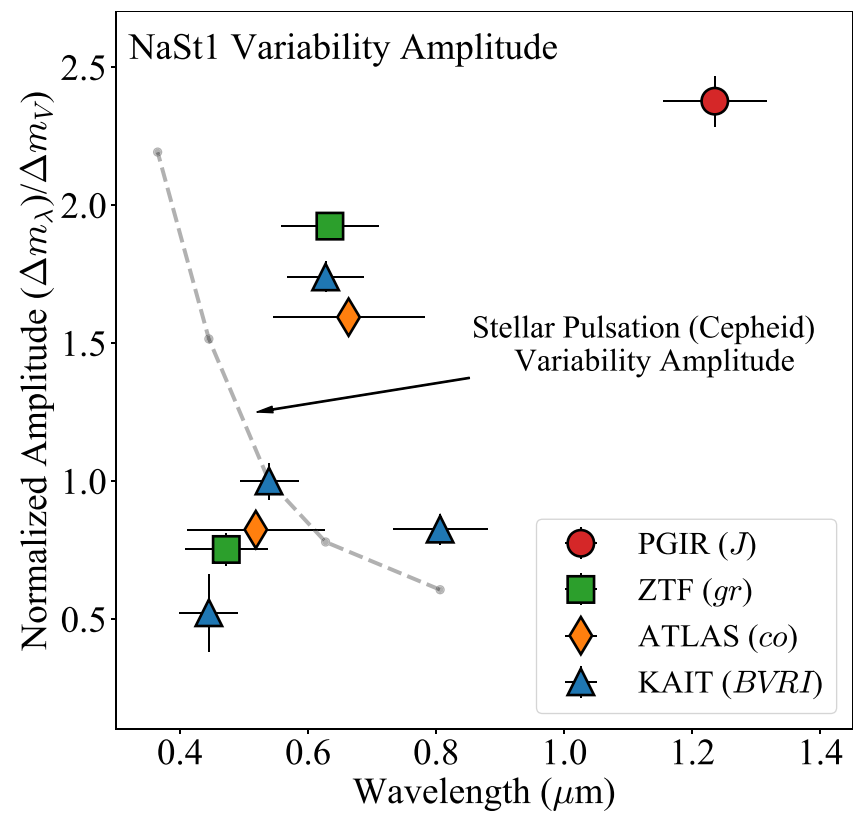

Figure 2. Normalized NaSt1 variability amplitudes from the adopted-period fits to the PGIR, ZTF, ATLAS, and KAIT light curves. The variability amplitudes, $\Delta m_{\lambda}$, observed from each filter with effective wavelength $\lambda$ are normalized to the KAIT $V$-band variability amplitude, $\Delta m_{V}=0.066 \pm 0.004$ mag. Horizontal error bars correspond to the filter bandwidths. The normalized variability amplitudes observed from stellar pulsations by Cepheids with long periods (log $P>1.02 ; \mathrm{Klagyivik} \&$ Szabados 2009) are overlaid on the plot.
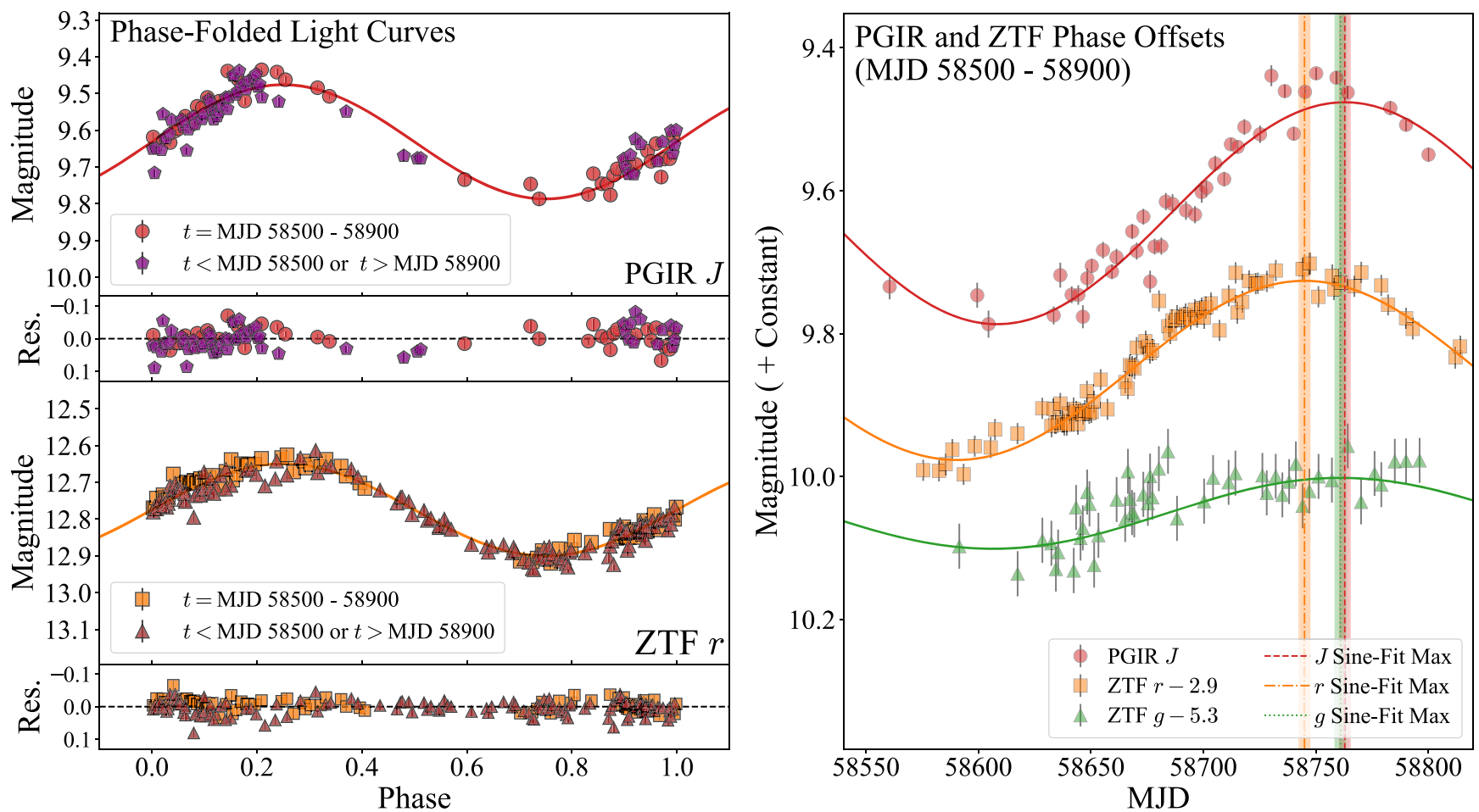

Figure 3. (Left:) Phase-folded PGIR $J$-band and ZTF $r$-band photometry overlaid with adopted-period sinusoidal light-curve fits. Residuals of the light curve are shown below each panel and demonstrate close agreement with sinusoidal variability. Observations obtained in the range MJD 58,500-58,900 are shown as red circles and orange squares for PGIR and ZTF, respectively. Observations obtained before MJD 58,500 or after MJD 58,900 are shown as purple pentagons or brown triangles for PGIR and ZTF, respectively. (Right:) Zoom-in of the PGIR $J$-band and ZTF $r$ - and $g$-band photometry between MJD 58,500 and MJD 58,900 overlaid with the adopted-period fits. Dates of the brightness maxima of each sinusoidal fit are overlaid as vertical lines with the corresponding uncertainties as shaded bands. The offset of the ZTF $r$-band maximum demonstrates the $17.0 \pm 2.5$ day phase offset from the ZTF $g$ - and PGIR $J$-band light-curve fits.

\subsection{Historical IR Variability}

Adopted-period $(P=309.7$ day $)$ light-curve fits to the UKIRT and ESO $1 \mathrm{~m} J H K L^{\prime}$-band observations of NaSt1 taken in 1983-1989 reveal that the periodicity of the past photometric variability is consistent with the present-day observations (Figure 4). Adopted-period light-curve fits to the $M$-band observations were unsatisfactory, which was likely due to the larger photometric uncertainties. Figure 4 also shows the predicted $J$-band emission based on the adopted-period fit to 
Table 4

NaSt1 Historical IR Light-Curve Fit Results

\begin{tabular}{lccc}
\hline \hline Filter & Amplitude (mag) & Mag. Offset & MJD $(\varphi=0)$ \\
\hline UKIRT/ESO $J$ U: $1.24(0.23) \mu \mathrm{m}$ E: $1.21(0.19) \mu \mathrm{m}$ & $0.25 \pm 0.02$ & $9.25 \pm 0.02$ & $45,607.8 \pm 7.0$ \\
UKIRT/ESO $H$ U: $1.65(0.26) \mu \mathrm{m}$ E: $1.64(0.27) \mu \mathrm{m}$ & $0.33 \pm 0.02$ & $8.18 \pm 0.02$ & $45,636.8 \pm 3.7$ \\
UKIRT/ESO $K$ U: $2.18(0.37) \mu \mathrm{m}$ E: $2.22(0.36) \mu \mathrm{m}$ & $0.29 \pm 0.02$ & $3.17 \pm 0.02$ & $45,633.4 \pm 3.9$ \\
UKIRT/ESO $L^{\prime}$ U: $3.77(0.53) \mu \mathrm{m}$ E: $3.74(0.58) \mu \mathrm{m}$ & $0.18 \pm 0.02$ & $3.87 \pm 0.02$ & $45,634.4 \pm 7.9$
\end{tabular}

Note. Instrument filter details (effective wavelength and bandwidth) and results from adopted-period $\left(P=309.7\right.$ day) sine fits to the historical $J H K L^{\prime}$-band light curves of NaSt 1 observed by UKIRT and ESO $1 \mathrm{~m}$. Fitted properties include the variability amplitude, magnitude offset at the mid-line of the fitted sine function, and the MJD where the phase of the sine function $\varphi=0$. The phase fitting assumed an initial $\varphi=0$ estimate of MJD 45,630.

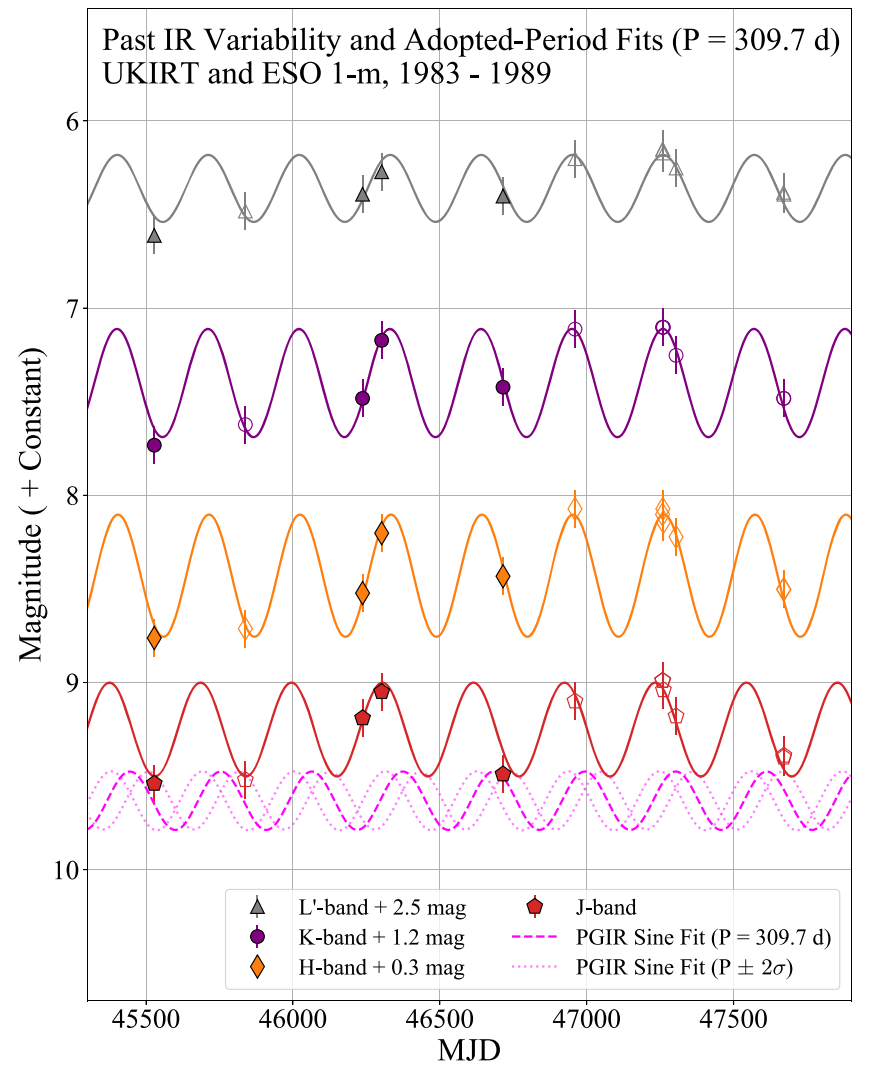

Figure 4. Adopted-period light-curve fits to $J H K L^{\prime}$-band photometry of NaSt1 observed from UKIRT (filled markers) and the ESO $1 \mathrm{~m}$ telescope (open markers) in the years 1983-1989 (MJD 45,528-47,671). The extrapolated photometric variability from the PGIR $J$-band adopted-period light-curve fits with $\pm 2 \sigma$ period uncertainties are plotted (in magenta).

the PGIR light curve. Note that despite the small uncertainties in the period ( $1 \sigma=0.6$ day) used for the light-curve fitting, the extrapolation from MJD 58,600 to MJD 46,500 leads to large uncertainties in the predicted phase. The consistent fit to the historic photometry strengthens our adopted period and demonstrates coherence of the periodic variability over decades.

The results from the adopted-period fits to the historical IR light curves are shown in Table 4. Similar to the results from the recent optical/IR light-curve analysis (Table 3), the variability amplitudes differ at different filter wavelengths. Interestingly, the results from the light-curve fits indicate that the past $J$-band emission was brighter and showed variability amplitudes larger than the recent PGIR $J$-band observations.

It is important to consider the color and magnitude conversions between the different IR photometric systems of
UKIRT/ESO and PGIR. Based on the UKIRT and 2MASS transformation Equations (37)-(40) from Carpenter (2001) and the measured magnitude offsets from the adopted-period lightcurve fits (Table 4), the 2MASS and UKIRT $J$-band photometry conversion is $J_{\text {UKIRT }}=J_{2 \text { MASS }}-(0.21 \pm 0.06 \mathrm{mag})$. Owing to their similar filter properties, $J_{2 \mathrm{MASS}} \approx J_{\mathrm{PGIR}}$, which implies that the change in $J_{\mathrm{PGIR}}$ between the past and recent observations is $\Delta J_{\mathrm{PGIR}}=0.17 \pm 0.06 \mathrm{mag}$. This suggests that NaSt1 does indeed exhibit variability on longer ( $\gtrsim$ decade) timescales.

\subsection{Mid-IR Variability}

Mid-IR spectroscopy of NaSt1 with BASS taken in 2017-2019 reveals variability at 3-13 $\mu \mathrm{m}$ (Figure 5), where the mid-IR flux from NaSt1 is likely dominated by thermal emission from circumstellar dust (Crowther \& Smith 1999; Rajagopal et al. 2007). The IR-dominated spectral-energy distribution (SED) in Figure 5 (left) also demonstrates that NaSt1 is enshrouded by circumstellar dust in addition to being obscured by interstellar material.

Figure 5 (center) shows the semicontemporaneous ZTF $g r$, ATLAS $c o$, and PGIR $J$ photometry taken within 20 days $(\Delta \varphi \approx 0.06)$ of the BASS spectroscopy in 2019 April (MJD $58,579)$ and within 7 days $(\Delta \varphi \approx 0.02)$ of BASS spectroscopy in 2019 August (MJD 58,719). Figure 5 (center) also shows the ZTF and ATLAS photometry taken within 4 days $(\Delta \varphi \approx 0.01)$ of the BASS spectroscopy in 2018 May (MJD 58,251). The timing of the 2019 April $(\varphi=0.71)$ and 2019 August $(\varphi=0.17)$ observations sample near the NaSt1 minimum and peak emission, respectively (see Figure 1). The NaSt1 SED at these two epochs shows that the trend in the mid-IR variability is consistent with the optical/near-IR variability (Figure 5, right). The ZTF, ATLAS, and PGIR photometry increased by $10 \%-$ 30\% between the 2019 April and 2019 August. The 3-13 $\mu \mathrm{m}$ emission increased similarly by $\sim 10 \%-30 \%$ between 2019 April and 2019 August, where the largest variations $(>20 \%)$ were observed at shorter mid-IR wavelengths $(\lambda \approx 3 \mu \mathrm{m})$. Variability from the circumstellar dust emission in the mid-IR is therefore likely linked to the optical/near-IR variability.

\subsection{Color Variability}

In order to investigate the color variability of NaSt1, KAIT, and ZTF/PGIR color curves were produced with simultaneous or nearly contemporaneous observations. Figure 6 (left) presents KAIT $V-I, V-R$, and $R-I$ color curves, which are derived directly from the simultaneous multiband KAIT observations. Since PGIR and ZTF observations were not performed simultaneously, the PGIR/ZTF $g-J, g-r$, and $r-J$ light curves in Figure 6 (right) were produced by utilizing observations taken within $<3$ days $(\Delta \varphi<0.01)$. 

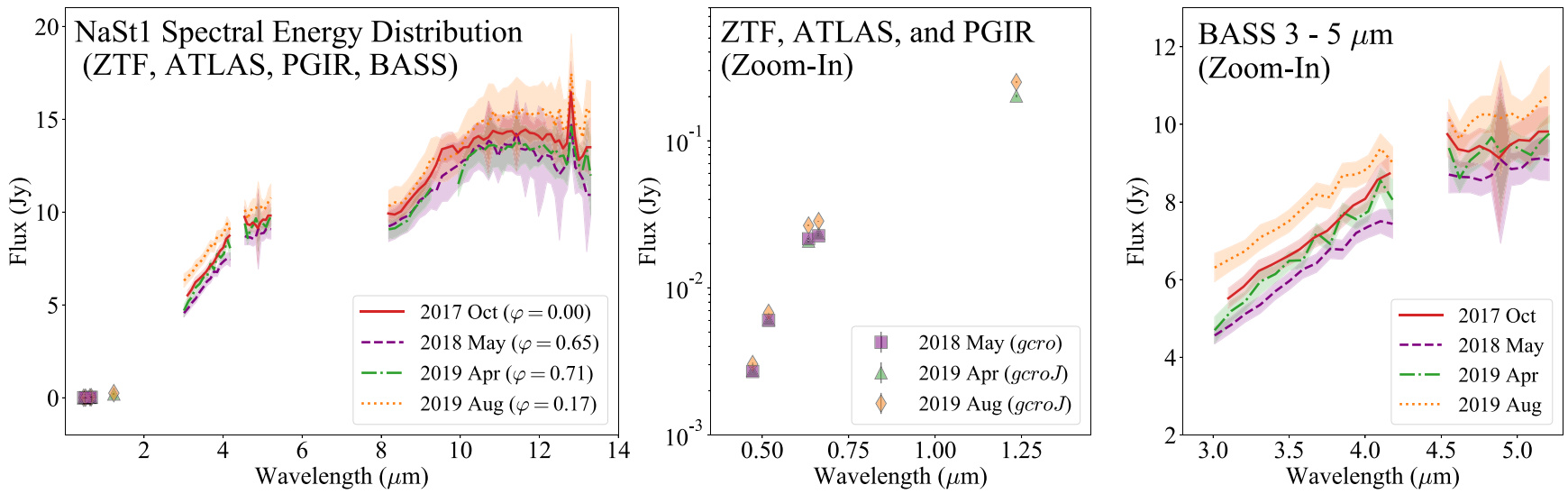

Figure 5. (Left) NaSt1 spectral-energy distribution variability demonstrated by four epochs of BASS mid-IR 3-13 $\mu$ m low-resolution spectroscopy and 3 epochs of ZTF $g r$-band, ATLAS co-band, and/or PGIR $J$-band photometric observations. BASS observations were taken in 2017 October (MJD 58,049, $\varphi=0$ ), 2018 May (MJD 58,251, $\varphi=0.65$ ), 2019 April (MJD 58,579, $\varphi=0.71$ ), and 2019 August (MJD 58,719, $\varphi=0.17$ ). Shaded regions correspond to $1 \sigma$ flux uncertainties. (Center) Zoom-in of the semi-contemporaneous ZTF, ATLAS, and PGIR observations. (Right) Zoom-in of 3-5 $\mu$ m region of the BASS observations that exhibit the mid-IR variability. Note that the zoom-in of the ZTF, ATLAS, and PGIR photometry is plotted on a semi-logarithmic scale, whereas the other plots are shown on a linear scale.
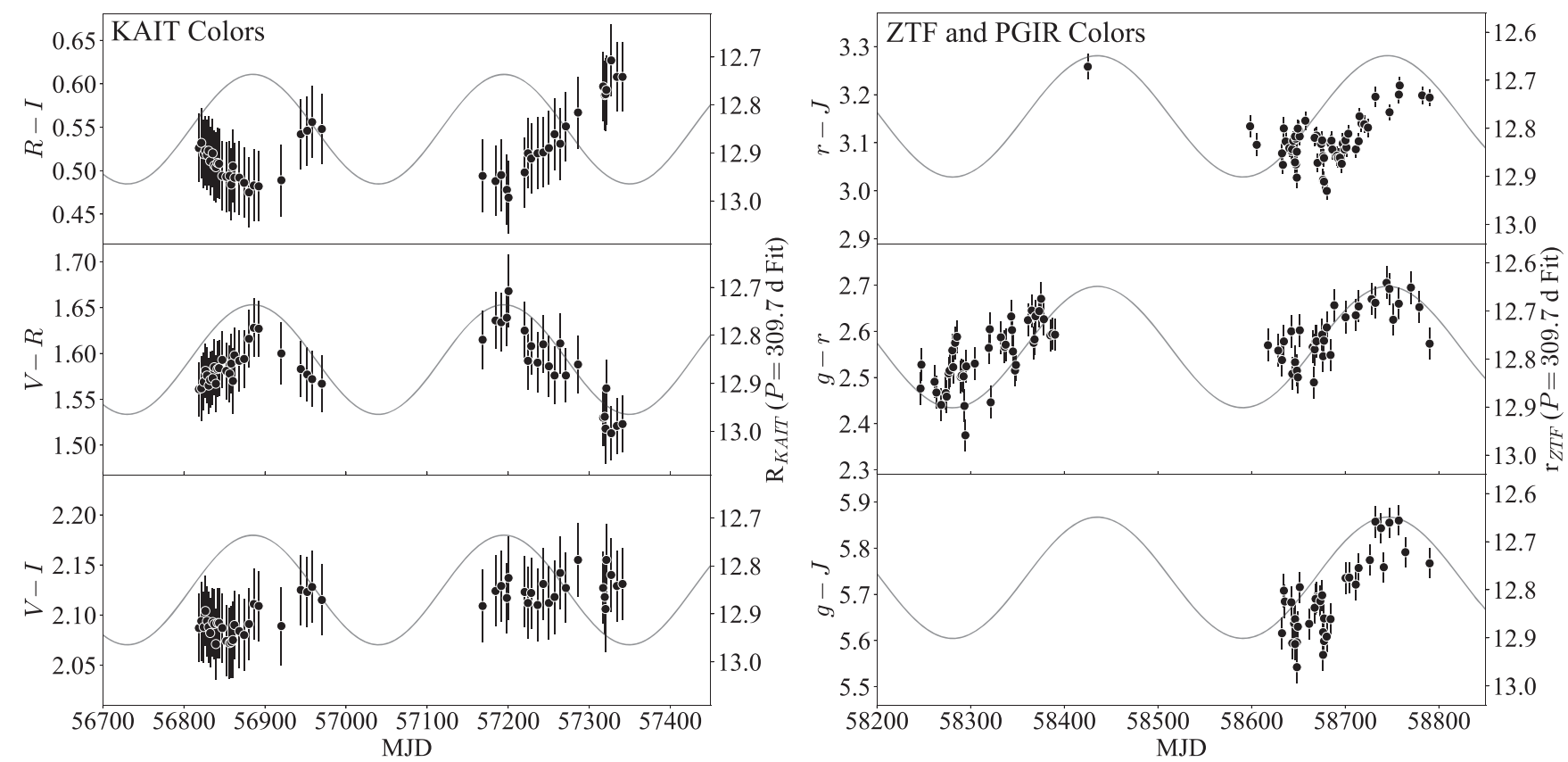

Figure 6. (Left) KAIT $V-I, V-R$, and $R-I$ colors between MJD 56,800 and MJD 57,350 overlaid with the KAIT $R$-band adopted-period sinusoidal model light curve. (Right) ZTF and PGIR $g-J, g-r$, and $r-J$ colors between MJD 58,200 and MJD 58,800 overlaid with the ZTF $r$-band light-curve fit.

KAIT color curves in Figure 6 (left) each show different variability trends. The $V-R$ color curve varied consistently with the $R$-band brightness; however, the $R-I$ curve indicates the opposite trend. The $V-I$ color curve exhibits no significant variability, which is likely due to the lower amplitude of the $I$-band variability relative to that of the shorter wavelength $R$-band filter (Figure 2). The ZTF and PGIR $g-J, g-r$, and $r-J$ color curves all show variability correlated with increasing $r$-band brightness. The discrepancies in the color evolution reflect the inconsistency of the variability amplitude from NaSt1 as a function of wavelength and suggest that different wavelength filters trace emission from different stellar and/or circumstellar components. The observed phase offsets between different filters (Figure 3, right) likely influence the color evolution as well.

\subsection{Emission-line Contributions}

Optical and near-IR spectroscopy of NaSt 1 by Crowther \& Smith (1999) revealed prominent narrow nebular emission lines such as $\mathrm{He} \mathrm{I} \lambda 6678, \mathrm{H} \alpha$, and [N II] $\lambda 5755$, which may contribute to the photometric measurements and variability. In order to determine whether the emission lines are influencing the variability, the NaSt1 emission-line list from Crowther \& Smith (1999) was used to quantify the relative observed fluxdensity contribution to the model-derived photometry (i.e., "Mag. Offset" in Table 3). A $10 \%$ absolute flux-calibration uncertainty was assumed for both the emission-line measurements from Crowther \& Smith (1999) and the PGIR, KAIT, ATLAS, ZTF, and ASAS-SN photometry.

Table 3 shows $F_{\text {line }} / F_{\text {phot }}$, the ratio of the flux density from NaSt1's observed emission lines over the filter bandwidths 


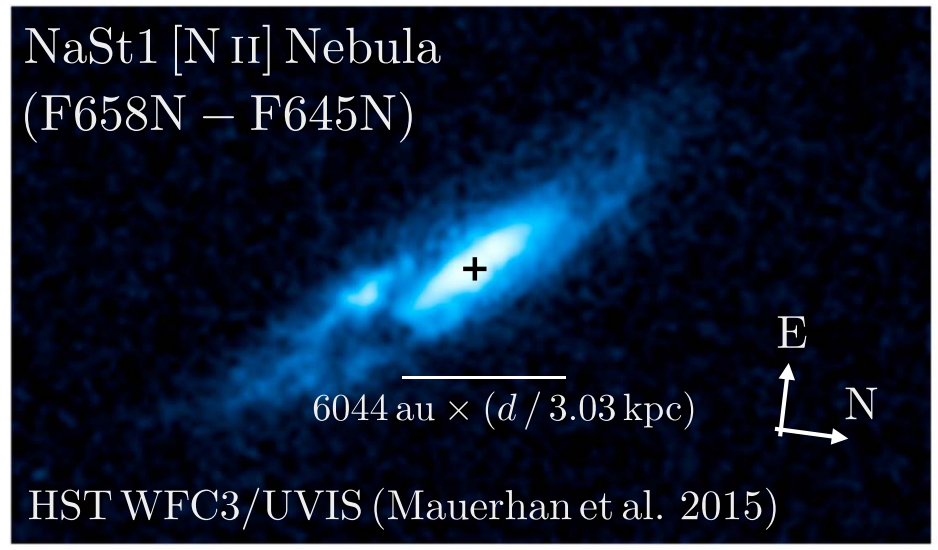

\section{NaSt1 Central Binary}

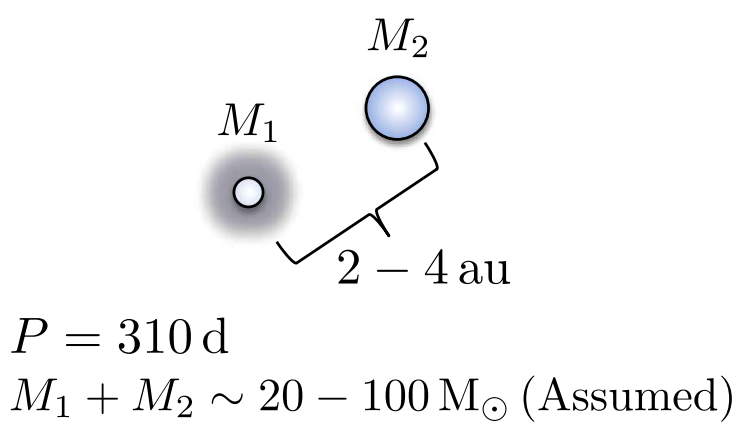

\section{Possible Binary Origins of Variability in NaSt1}

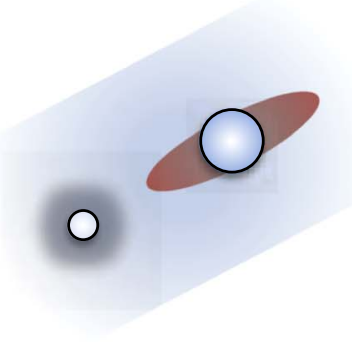

Disk Eclipses

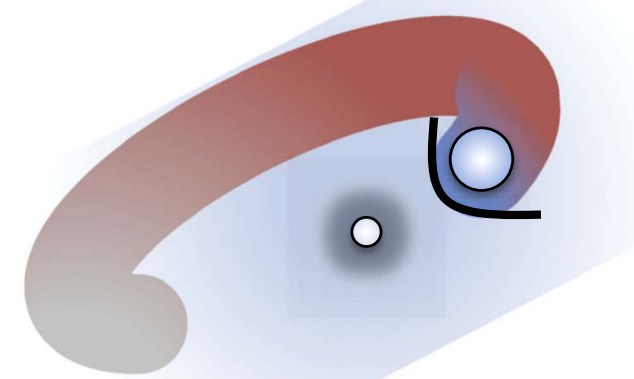

Colliding-Wind Dust Formation
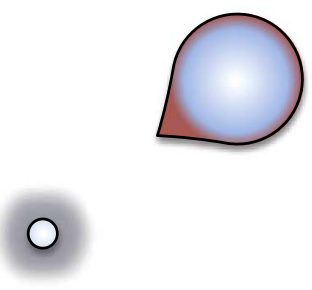

Ellipsoidal Variability

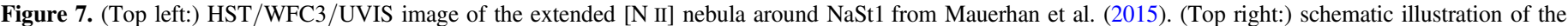

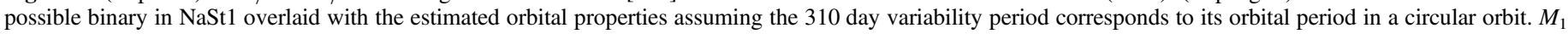

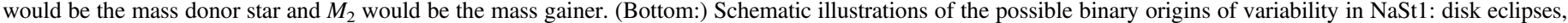
colliding-wind dust formation, and ellipsoidal variability. Note that the illustrations in this figure are not drawn to scale.

relative to the observed photometry. The nebular emission lines from NaSt1 do indeed appear to contribute $\sim 5 \%-30 \%$ to the optical photometry, where the strongest emission lines in $\mathrm{NaSt} 1$ are associated with He I, He II, and [N II]. However, the emission-line contribution is much lower in $J$. Since the amplitudes in the optical light curves are in the range $\sim 0.03-0.13 \mathrm{mag}$, or $\sim \pm 3 \%-11 \%$ of the "Mag. Offset," it is possible that the photometric variability may be due to changes in the observed strength of the emission lines in these filters. Interestingly, the ATLAS $o$, ZTF $r$, and KAIT $R$ observations which exhibit the largest photometric variability amplitudes at optical wavelengths (Figure 2) are the only bands that include coverage of He I $\lambda 6678$, the strongest optical emission line observed from NaSt1 (Crowther \& Smith 1999). These are also the only filter bands that include coverage of $\mathrm{H} \alpha$ and [N II] $\lambda 6583$, where emission from the latter appears as an extended and asymmetric nebula around NaSt1 (Mauerhan et al. 2015; Figure 7, top)

The emission-line contribution to the PGIR $J$-band photometry is negligible, which indicates that the large $J$ variability amplitude does not arise from variable near-IR emission lines. There is also minimal $\left(F_{\text {line }} / F_{\text {phot }}<0.01\right)$ emission-line contribution to the past $J H K$ photometry from UKIRT/ESO (Table 4), which suggests that the apparent change in the $J$ band amplitude variability between the UKIRT/ESO and PGIR observations is not due to emission-line variations. $J$-band emission from NaSt1 may instead be dominated by thermal emission from circumstellar dust.

It is possible that the PGIR $J$, ATLAS $o$, ZTF $r$, and KAIT $R$ bands exhibit high-amplitude variability because the filter bands trace emission with a significant contribution from circumstellar material. The other filter bands with lower variability amplitudes may instead be more influenced by stellar continuum emission.

\section{Discussion}

\subsection{Possible Single-Star Origins of Variability}

NaSt1's asymmetric nebula (Figure 7, top; Mauerhan et al. 2015) combined with the discovery of its regular period suggests that NaSt1 hosts a binary. Photometric variability arising from pulsations or other intrinsic instability in hot, single, massive stars also do not typically appear as regular or sinusoidal as we observe from NaStl (e.g., Soraisam et al. 2020). We therefore postulate that the smooth 310 day photometric variability is most likely modulated by the orbit of a binary in NaSt1. This discussion is notably reminiscent of the long debate on the binary versus single-star hypotheses on $\eta$ Car, where the binary interpretation is favored based on observations of its stable periodic variability (Damineli 1996; Corcoran 2005; Damineli et al. 2008). However, it is still 
important to consider the possible single-star mechanisms that may also account for the observed variability in NaSt 1 .

One of the most common origins of stellar variability is stellar pulsations. This mechanism can result in near-sinusoidal photometric variations, which have been observed from stars such as Cepheids, Miras, and red supergiants (RSGs). However, the amplitude of the photometric variability arising from stellar pulsations exhibits a characteristic wavelength dependence where the amplitudes increase toward shorter wavelengths owing to associated changes in the star's effective temperature (e.g., Klagyivik \& Szabados 2009). This is inconsistent with the observed variability amplitudes of NaSt1 at different wavelength filters as shown in Figure 2. We therefore argue against stellar pulsations as the origin of NaSt1's photometric variability.

Another possibility for a single-star variability origin is rotational modulation that occurs in a rare class of highly magnetized O stars with the peculiar "Of?p" classification (Walborn 1973; Howarth et al. 2007; Grunhut et al. 2017). Of? p stars exhibit near-sinusoidal variability on the order of $\sim 0.05$ mag and are attributed to phase-dependent occulations of a wind-trapped magnetosphere (Nazé et al. 2015; Munoz et al. 2020). They can also be slow rotators with rotation periods that range from weeks to years (Nazé et al. 2015). Of?p stars also exhibit dramatic spectral variability. For example, the wellknown Galactic Of?p star HD 191612 exhibits both photometric variations and strongly variable Balmer and He I lines on a 540 day period (Howarth et al. 2007). Interestingly, another Galactic Of?p star, HD 148937, is surrounded by an extended dusty and N-rich circumstellar nebula (Mahy et al. 2017), which is similar to the properties of NaSt1's nebula. The photometric variability of Of?p stars, however, is modulated by wavelength-independent electron scattering opacity from a wind-trapped magnetosphere (Munoz et al. 2020). The variability amplitudes should therefore show no significant deviations as a function of wavelength. Howarth et al. (2007) also show that the photometric variations in HD 191612 must arise from continuum-level variability rather than changes in the line emission strength. Although it is unlikely, we cannot completely rule out the Of?p phenomenon as a contributor to NaSt1's photometric variability.

\subsection{Possible Binary Origins of Variability}

Given our discussion in the previous section, we assume that NaSt1's photometric variability is associated with the orbital period of a binary system as opposed to a single-star origin. Here, we examine the possible binary origins of NaSt1's variability and the relation to its orbital period. First, we can infer some orbital properties of the binary system assuming that the orbital period is consistent with the observed 310 day variability period. Assuming a combined stellar mass in the range $\sim 20-100 M_{\odot}$ for the binary and a circular orbit, a 310 day orbital period corresponds to an orbital separation of $\sim 2-4$ au. We note that a circular orbit is expected for a system that recently underwent RLOF. The orientation of the binary system can be inferred from NaSt1's extended N-rich nebula, which exhibits a nearly edge-on geometry with an inclination angle of $i=78^{\circ} \pm 3^{\circ}$ (Mauerhan et al. 2015). Figure 7 (top) shows the resolved HST imaging by Mauerhan et al. (2015) of NaSt1's N-rich nebula and our schematic illustration of the embedded central binary. In this section, we discuss three possible binary-related scenarios given $\mathrm{NaSt1}$ 's 310 day period and smooth sinusoidal variations (Figure 7, bottom): disk eclipses, colliding-wind dust formation, and ellipsoidal variability. We acknowledge that other scenarios are also possible given the limited observational constraints, but for the sake of brevity they will not be considered here.

\subsubsection{Disk Eclipses}

Given a large orbital separation ranging over several au, it is highly unlikely that the smooth, sinusoidal variability could arise from eclipses from the stars themselves, which would instead appear as narrow eclipses. However, we suggest that the variability may be caused by eclipses of a circumbinary disk from an optically and geometrically thick circumstellar disk in NaSt1 that formed as a result of the RLOF mass-transfer process. Several prominent emission lines in NaSt1 (e.g., [N II] 15755; Crowther \& Smith 1999) show double-peaked profiles that indeed indicate the presence of a disk.

This scenario is similar to the massive eclipsing binary RY Scuti, which is currently undergoing RLOF with a mass gainer that appears to be enshrouded by an optically and geometrically thick accretion disk (Grundstrom et al. 2007; Djurašević et al. 2008; Smith et al. 2011b). RY Scuti also exhibits smooth, periodic variability; however, its 11 day orbital period is much shorter than that of $\mathrm{NaSt1}$, and its variability is not as consistent with a sine wave (Djurašević et al. 2008).

Given the significant contribution from the strong nebular emission lines to the observed photometry in NaSt1 (Table 3), it is possible that the photometric variability is linked to the obscuration of line-emitting regions from a circumbinary disk by a circumstellar disk. Optical variability in this scenario could arise from a circumstellar disk obscuring a varying fraction of the emitting surface area of a circumbinary disk, which requires the density distribution of the circumbinary disk to deviate from circular symmetry. This may be expected if circumbinary material is associated with NaSt1's asymmetric [N II] nebula (Figure 7). The observed IR variability may then arise from disk eclipses of the observed circumstellar/ circumbinary dust component around NaSt1 with an extent of $\lesssim 150$ au (Rajagopal et al. 2007). ${ }^{21}$ A similar alternative scenario is that a circumstellar disk may block a significant fraction of the ionizing and dust-heating radiation field from the stripped-envelope, mass-donor star. If the surrounding nebulae of dust and ionized gas are asymmetric, this scenario could also lead to optical/IR variability throughout the orbit of the central binary.

Another disk-related scenario, where the mass gainer in NaSt1 has an optically thick accretion disk like RY Scuti, is that the photometric variability may arise from the disk precession. The emission from the disk could be powered by reprocessed light from the hot, ionizing star in NaSt1, which would be analogous to the 162 day "super-orbital" period from the Galactic microquasar SS 433 (e.g., Fabrika 2004). However, such a scenario should present complex photometric variability modulated by a shorter-timescale orbital period. Although a detailed analysis of this hypothesis is beyond the scope of this work, we consider this scenario unlikely given NaSt1's smooth and stable sinusoidal variability.

\footnotetext{
21 This has been updated with the recent $d=3.03 \mathrm{kpc}$ distance estimate to NaSt1 (Rate \& Crowther 2020), and we note that Rajagopal et al. (2007) had adopted a distance of $2 \mathrm{kpc}$.
} 


\subsubsection{Colliding-wind Dust Formation}

Mid-IR and X-ray observations of NaSt1 indicate the presence of ongoing or recent dust formation that may be due to colliding winds (Rajagopal et al. 2007; Mauerhan et al. 2015). Dust formation via colliding winds is common in latetype carbon-rich WR (WC) binaries (Williams et al. 1987) and is also believed to occur in $\eta$ Car (Smith 2010). In this process, the strong wind from the WR star collides with the weaker wind from an OB-star companion which creates a dense shock front that cools and forms dust in the wake of the OB star (Usov 1991; Tuthill et al. 2008). An inclined dust-forming WR binary can exhibit smooth IR variability owing to viewing perspective variations toward the dust-forming region throughout the system's orbit (Monnier et al. 1999; Hendrix et al. 2016). The line-of-sight optical depth to the dust-forming regions can vary with viewing angle due to obscuration from optically thick regions of the colliding-wind shock cone. These effects are evident in the colliding-wind dust-forming WR system WR 98a (WC8-9vd; Williams et al. 1995), which exhibits a "rotating" dusty pinwheel nebula and roughly sinusoidal IR variability that are both consistent with a 565 day period (Monnier et al. 1999). HD 36402, a WC4 $(+\mathrm{O} ?)+\mathrm{O} 8 \mathrm{I}$ system in the Large Magellanic Cloud (Moffat et al. 1990), also exhibits dust formation and sinusoidal IR variability with a $5.1 \mathrm{yr}$ photometric period (Williams et al. 2013; Williams 2019).

Observations of NaSt1 do not present an obvious dusty pinwheel nebula; however, a dusty pinwheel may be difficult to resolve owing to its shorter orbital period and high inclination. It is therefore plausible that the near-IR and mid-IR variability from NaSt1 may be due to a mechanism similar to that seen in WR 98a and HD 36402.

In the colliding-wind dust-formation scenario, the optical variability observed from NaSt 1 could be linked to obscuration of a circumbinary nebula by optically thick regions of the colliding-wind shock cone and/or newly formed dust. As mentioned in Section 3.5, the variability amplitudes of the optical and IR light curves may therefore be associated with the fraction of the circumstellar/circumbinary emission over the stellar continuum emission in each filter band. The filter bands that exhibit the highest variability amplitude are notably the PGIR $J$ band, which should be dominated by circumstellar dust, and the ZTF $r$, KAIT $R$, and ATLAS $o$ bands, which cover the brightest nebular emission line He I $\lambda 6678$ as well as $\mathrm{H} \alpha$ and $[\mathrm{N} \mathrm{II}] \lambda 6583$. The other filter bands that show lower amplitude variability are less influenced by the nebular emission and may capture variability influenced by the stellar continuum emission.

We note that it is unlikely that the observed variability of $\mathrm{NaSt} 1$ arises from variable or episodic dust formation since we would expect anti-correlated IR and optical light curves, where newly forming IR-luminous dust obscures the optical emission.

\subsubsection{Ellipsoidal Variability}

Given the interpretation of binary mass transfer in NaSt1 (Mauerhan et al. 2015), the stars and/or a circumstellar disk may be tidally distorted and exhibit ellipsoidal variability as the system reveals different cross sections throughout its nearly edge-on orbit (e.g., Morris \& Naftilan 1993). Such variations may naturally reproduce some of the wavelength-dependent amplitudes of the light curve owing to temperature gradients along the surface of the elongated star or disk.

It is important to note that if the NaSt1 light curve was modulated by ellipsoidal variability throughout a binary orbit, its orbital period would be twice that of the period derived from the sinusoidal model because two consecutive brightness peaks would correspond to the two phases of quadrature in a full orbit. Tidally elongated stars in such a wide orbital configuration would therefore have to exhibit large radii. Assuming a $2 \times 310=620$ day orbital period, a combined stellar mass range of $20-100 M_{\odot}$, and a binary mass ratio of $0.25-4.0$, the radius of the tidally distorted star would have to approach its Roche-lobe radius of $220-710 R_{\odot}$ (Eggleton 1983). In this scenario, the tidally distorted star should therefore possess a radius consistent with that of a supergiant.

\subsubsection{Preferred Interpretation and Unresolved Issues}

Given the evidence for dust formation, we favor the interpretation that NaSt1's periodic variability is linked to variable line-of-sight optical depth effects from colliding-wind dust formation. The longer timescale variability suggested by the past $J$-band light curve (Figure 4) may be a product of stellar variability and/or fluctuations in the optical/UV radiation heating the circumstellar dust. We cannot conclusively rule out a possible contribution to NaSt1's periodic photometric variability from disk eclipses and/or ellipsoidal variability. In Sections 4.3 and 4.4, we will argue that NaSt1 does not host a supergiant, which suggests that ellipsoidal variability from a tidally distorted star is unlikely.

It is interesting to note that almost all known massive dustforming colliding-wind binaries exhibit a C-rich chemistry and host a WC star (Crowther 2007). Future work on determining the composition of circumstellar dust around $\mathrm{NaSt} 1$ will be important for distinguishing the chemistry of the enshrouded star(s).

Unfortunately, it is difficult to deduce a comprehensive interpretation of NaStl's variability that satisfies all of the observational constraints. Most notably, there are still unresolved issues on how to account for the apparent phase delay between different filters (Figure 3, right; Table 3) as well as the origin of longer timescale variability suggested by the historical $J$-band light curve (Figure 4). We can speculate on the origin of the 17 day phase offset between the PGIR $J$ and ZTF $r$ light curves (Figure 3, right), where the phase-delay timescale corresponds to a light-travel distance of $2940 \mathrm{au}$, or 1 ". 0 at a distance of $3.03 \mathrm{kpc}$. Given the $\sim 1^{\prime \prime}$ extent of bright circumstellar material in the central regions of NaSt1's nebula (Figure 7, top left), it is possible that the phase delay corresponds to the light-travel time between stellar and/or circumstellar components of NaSt1.

Owing to the complexity and obscured nature of NaSt1, further observations and theoretical modeling are needed to resolve these issues and test our hypotheses. Three-dimensional radiative transfer models would be valuable for exploring how variations in line-of-sight optical depths from colliding-wind dust formation affect the optical and IR light curves as well as the multi-epoch mid-IR BASS spectra. Continued multiwavelength photometric as well as spectroscopic monitoring of NaSt1 will also be important for resolving the origin(s) of its variability. 


\subsection{On the Nature of NaSt1: An Ofpe/WN9 Eruption?}

Assuming that the photometric variability is modulated by the orbital motion of a binary in NaSt1, we can speculate on its nature in the context of the mass-transfer interpretation presented by Mauerhan et al. (2015). The $\sim 2-4$ au binary orbital separation (Section 4.1) allows us to estimate the Rochelobe radius that the mass-donor star must have approached in order to trigger mass transfer. Adopting a binary mass ratio of $0.25-4.0$, the Roche-lobe radius is in the range $140-450 R_{\odot}$. Although such a large stellar radius might suggest an RSG mass donor, an RSG is not consistent with the high temperatures ( $\gtrsim 30,000 \mathrm{~K})$ inferred from NaSt1's high-excitation nebular emission lines and there are no spectroscopic RSG features in the the IR where its emission should be directly observable (Crowther \& Smith 1999).

Crowther \& Smith (1999) suggested that the highly CNOprocessed composition of the nebula and the temperature of the hot ionizing source is consistent with an early-type nitrogenrich WR (WN) star; however, they demonstrated that NaSt1 does not exhibit any broad features consistent with an early WN star. It is important to note that the absence of spectroscopic confirmation of WR features does not preclude the presence of a WR star in NaSt1. The LBV system $\eta$ Car is also enshrouded by dense circumstellar material and hosts an unseen hot massive companion that is thought be a WR star (see Smith et al. 2018 and references therein).

Interestingly, Smith et al. (2020) presented observations of an Ofpe/WN9 star, which are stars that show properties intermediate between those of Of and WN stars (Walborn 1977; Bohannan \& Walborn 1989), and confirmed that these types of stars can undergo an LBV-like eruption. During this eruptive phase, the radius of the Ofpe/WN9 star can expand to double the size of its quiescent radius (Smith et al. 2020). Ofpe/WN9 stars in the Galactic center exhibit radii of $R_{2 / 3} \approx 35-80 R_{\odot}$, which corresponds to the radius where $\tau_{\text {Rosseland }}=2 / 3$ (Martins et al. 2007). We therefore suggest that mass transfer in $\mathrm{NaSt} 1$ could have been triggered during an eruptive phase from an Ofpe/WN9 star that is now obscured or outshined by dense circumbinary or circumstellar material. The massive RLOF binary RY Scuti is also believed to have undergone past LBV-like eruptions that led to the formation of its toroidal circumstellar nebula (Smith et al. 2011b).

Earlier, van der Hucht et al. (1989, 1997) had proposed that NaSt1 may host an Ofpe/WN9 star; however, this was based only on the nebular spectrum. Crowther \& Smith (1999) instead suggested that NaSt1 hosts an early-type WN star based on the high temperatures required for the ionization source of its nebula. Our Ofpe/WN9 hypothesis on the nature of NaSt1 is still consistent with the interpretation by Crowther \& Smith (1999): after its eruption, the mass donor may no longer resemble an Ofpe/WN9 star and could have transitioned to a hotter, earlier-type WN phase like the Ofpe/WN9 star MCA$1 B$ studied by Smith et al. (2020). It is also possible that envelope stripping may have led to a more advanced chemical composition than the WN phase such as the WC phase. However, the deficiency of carbon in the circumstellar environment around NaSt1 would require a very recent transition to a WC star.

We can obtain insight on the enshrouded star(s) in NaSt1 from radio observations taken by the Karl G. Jansky Very Large Array telescope in the Global View of Star Formation in the Milky Way (GLOSTAR) survey (Medina et al. 2019). Interestingly,
NaSt1 exhibits a radio spectral index of $\alpha=0.61 \pm 0.10$ between $4 \mathrm{Ghz}$ and $8 \mathrm{GHz}$, consistent with the spectral index expected for free-free emission from an ionized stellar wind $(\alpha \approx 0.6$; Panagia \& Felli 1975; Wright \& Barlow 1975). Such thermal radio emission properties are commonly identified from the ionized winds of OB and WR stars (Abbott et al. 1986; Bieging et al. 1989). We can then estimate the mass-loss rate of the ionized wind given the theoretical relation between the observed radioflux density $F_{\nu}$ and the stellar-wind parameters (Wright \& Barlow 1975):

$$
F_{\nu}=2.32 \times 10^{4}\left(\frac{\dot{M} Z}{v_{\infty} \mu}\right)^{4 / 3}\left(\frac{\gamma g_{\nu} \nu}{d^{3}}\right)^{2 / 3} \mathrm{mJy},
$$

where $\dot{M}$ is the mass-loss rate in $M_{\odot} \mathrm{yr}^{-1}, v_{\infty}$ is the wind velocity in $\mathrm{km} \mathrm{s}^{-1}, \mu$ is the mean molecular weight, $Z$ is the mean ionic charge, $\gamma$ is the number of electrons per ion, $g_{\nu}$ is the Gaunt factor at observed frequency $\nu$, and $d$ is the distance in kpc. In order to estimate the mass-loss rate from the NaSt1 radio counterpart, we assume that $\mu \approx 4.0$, which is consistent with H-poor winds, and $Z=1.1, \gamma=1.1$, and $g_{5.8} \approx 5.0$ (Leitherer et al. 1997). Given the absence of any obvious stellar emission features from NaSt1's optical and near-IR spectrum (Crowther \& Smith 1999), we must provide a guess for the wind velocity associated with the radio counterpart. Since the wind is most likely ionized by a hot central WR or OB star, we adopt a wind velocity of $1000 \mathrm{~km} \mathrm{~s}^{-1}$. From NaSt1's observed $5.8 \mathrm{GHz}$ flux density of $F_{5.8 \mathrm{GHz}}=4.38 \pm$ $0.23 \mathrm{mJy}$ (Medina et al. 2019), we then estimate a mass-loss rate of

$$
\dot{M} \approx 2 \times 10^{-4}\left(\frac{v_{\infty}}{1000 \mathrm{~km} \mathrm{~s}^{-1}}\right) M_{\odot} \mathrm{yr}^{-1} .
$$

This mass-loss rate estimate is likely uncertain by factors of a few given NaSt1's unknown wind velocity. However, we note that a mass-loss rate of $\sim 10^{-4} M_{\odot} \mathrm{yr}^{-1}$ is consistent with the upper range of mass-loss rates exhibited by Galactic WN stars (Hamann et al. 2019).

\subsection{No (More) Mass Transfer in NaSt1?}

In this section, we address whether NaSt1 is undergoing RLOF mass transfer assuming the observed photometric variability is modulated by the orbital motion of the interacting (or previously interacting) binary. In order to assess the current state of mass transfer, we estimate the radius of the hot, ionizing star in NaSt 1 and compare it to the Roche-lobe radius $\left(R_{L} \approx 140-450 R_{\odot}\right)$ derived above.

First, we obtain an estimate of the stellar luminosity from NaSt1's observed brightness $V=14.4$ mag. By adopting the interstellar and/or circumstellar extinction correction toward NaSt1 by Crowther \& Smith (1999) of $A_{V}=6.5 \mathrm{mag}$ and using the Gaia-derived distance of $d=3.03 \mathrm{kpc}$ (Rate \& Crowther 2020), the absolute magnitude of NaSt1 is $M_{V}=-4.5$. The $30,000 \mathrm{~K}$ lower limit of the temperature derived for the ionizing source in NaSt1 by Crowther \& Smith (1999) from optical spectroscopy implies a bolometric correction is $B C_{V}=-3$ mag, which indicates a stellar luminosity of $L \approx 7.9 \times 10^{4} L_{\odot}$. The radius of such a $30,000 \mathrm{~K}$ star would be $R_{*} \approx 10 R_{\odot}$, an order of magnitude less than the estimated Roche-lobe radius. Stellar temperatures higher than $30,000 \mathrm{~K}$ will require larger 
Table 5

NaSt1 Properties Summary

\begin{tabular}{|c|c|c|}
\hline Property & Value & Ref \\
\hline $\begin{array}{l}\text { Equation Coordinates } \\
\qquad(\mathrm{J} 2000)\end{array}$ & $18^{\mathrm{h}} 52^{\mathrm{m}} 17^{\mathrm{s}} .55,+00^{\circ} 59^{\prime} 44^{\prime \prime} 3$ & 1 \\
\hline Gal. Coordinates (J2000) & $033.9154,+00^{\circ} .2639$ & 1 \\
\hline $\mathrm{PM} \mu_{\alpha} \cos \delta$ & $-0.271 \pm 0.080{\text { mas } \mathrm{yr}^{-1}}^{-1}$ & 1 \\
\hline $\mathrm{PM} \mu_{\delta}$ & $-0.614 \pm 0.074{\text { mas } \mathrm{yr}^{-1}}^{-1}$ & 1 \\
\hline Distance & $3.03_{-0.45}^{+0.60} \mathrm{kpc}$ & 2 \\
\hline Spec. Classifications & $\begin{array}{c}\text { WN10 Ofpe/WN9, B[e], O[e] Early } \\
\text { WR }+\eta \text { Car-like Nebula? }\end{array}$ & $\begin{array}{c}3,45 \\
67\end{array}$ \\
\hline$A_{V}$ & $6.5 \mathrm{mag}$ & 7 \\
\hline Var. Period & $309.7 \pm 0.7$ day & 8 \\
\hline$F_{5.8 \mathrm{GHz}}$ & $4.38 \pm 0.23 \mathrm{mJy}$ & 9 \\
\hline Spec. Index, $\alpha$ & $0.61 \pm 0.10$ & 9 \\
\hline$\dot{M}$ & $\sim 10^{-4} M_{\odot} \mathrm{yr}^{-1}$ & 8 \\
\hline$L_{X}$ & $\sim 10^{33} \mathrm{erg} \mathrm{s}^{-1}$ & 10 \\
\hline$L_{\mathrm{bol}}$ & $\sim 10^{5}-10^{6} L_{\odot}$ & 7,8 \\
\hline$B\left(\Delta m_{B}\right)$ & $16.208(0.034)$ & 8 \\
\hline$g\left(\Delta m_{g}\right)$ & $15.349(0.049)$ & 8 \\
\hline$c\left(\Delta m_{c}\right)$ & $14.370(0.054)$ & 8 \\
\hline$V\left(\Delta m_{V}\right)$ & $14.414(0.066)$ & 8 \\
\hline$R\left(\Delta m_{R}\right)$ & $12.850(0.114)$ & 8 \\
\hline$r\left(\Delta m_{r}\right)$ & $12.775(0.126)$ & 8 \\
\hline$o\left(\Delta m_{o}\right)$ & $12.608(0.105)$ & 8 \\
\hline$I\left(\Delta m_{I}\right)$ & $12.303(0.054)$ & 8 \\
\hline$J\left(\Delta m_{J}\right)$ & $9.632(0.156)$ & 8 \\
\hline$J_{\text {Hist }}\left(\Delta m_{J, \text { Hist }}\right)$ & $9.25(0.25)$ & 8 \\
\hline$H_{\text {Hist }}\left(\Delta m_{H, \text { Hist }}\right)$ & $8.18(0.33)$ & 8 \\
\hline$K_{\text {Hist }}\left(\Delta m_{K, \text { Hist }}\right)$ & $6.17(0.29)$ & 8 \\
\hline$L_{\text {Hist }}^{\prime}\left(\Delta m_{L^{\prime}, \text { Hist }}\right)$ & $3.87(0.18)$ & 8 \\
\hline
\end{tabular}

Note. References: 1-Gaia Collaboration (2018), 2-Rate \& Crowther (2020), 3-Nassau \& Stephenson (1963), 4-Massey \& Conti (1983), 5-van der Hucht et al. (1989), 6-van der Hucht et al. (1997), 7-Crowther \& Smith (1999), 8-This work, 9-Medina et al. (2019), 10-Mauerhan et al. (2015).

bolometric corrections and thus lead to higher stellar luminosities; however, the resulting radii will be smaller than that of the $30,000 \mathrm{~K}$ star. For example, a star with a temperature of $200,000 \mathrm{~K}$, which is the upper limit of the ionizing source temperature derived by Crowther \& Smith (1999), would imply a luminosity of $L \approx 3.1 \times 10^{6} L_{\odot}$ and a stellar radius of $R_{*} \approx 1.5 R_{\odot}$ given the appropriate bolometric correction of $B C_{V}=-7$ mag. Based on these constraints, we suggest that $\mathrm{NaSt} 1$ is not currently undergoing binary mass transfer.

\section{Conclusions}

A comprehensive summary of NaStl's properties including the results from our work are provided in Table 5. In this paper, we presented the discovery of a 310 day photometric variability period from NaSt1 with optical and near-IR light curves obtained between 2014 July and 2021 July (Figure 1). The best-constrained period, $P=309.7 \pm 0.7$ day, is derived from a sinusoidal fit to the ZTF $r$-band light curve. The amplitude of NaSt1's sinusoidal variability differed at different wavelengths, and we identify a significant phase shift between the sine models fit to the $r$-band and $J$-band light curves (Table 3; Figure 3, right). Based on previously cataloged optical emission lines from the nebula surrounding NaSt1 (Crowther \& Smith 1999), we determined that nebular emission lines contribute $\sim 5 \%-30 \%$ to the optical photometry (Table 3 ). The fraction of emission originating from circumstellar material may therefore be linked to the differing variability amplitudes observed across the filter wavelengths. Historical IR light curves over 1983-1989 show that the past variability was consistent with the present-day period of 310 days. However, the past $J$-band brightness and variability amplitudes (Table 4) were greater than that measured by the recent PGIR light curves, suggesting that NaStl exhibits variability on longer ( $\gtrsim$ decade) timescales. Mid-IR AEOS/BASS spectra of NaSt1, which were taken semi-contemporaneously with ZTF, ATLAS, and PGIR observations, demonstrated that its 3-13 $\mu \mathrm{m}$ emission is also variable and appears to be correlated with the optical/near-IR variability (Figure 5).

We discussed possible single-star origins for NaSt1's photometric variability. Stellar pulsations are an unlikely origin given the inconsistency between the observed and expected trend in the variability amplitude as a function of wavelength (see Figure 2). We also considered rotational modulation in a rare class of highly magnetized $\mathrm{O}$ stars (Of?p) as a possible origin of single-star variability. However, the amplitude of photometric variations from Of?p stars should show no significant deviations as a function of wavelength, which is also discrepant from the observations of NaSt 1 . We therefore postulate that the observed 310 day optical/near-IR periodic, sinusoidal variability is associated with the orbital period of a binary system in NaSt1. Although the properties of the stars in this binary system are still unknown, we use the 310 day orbital period to estimate an orbital separation of $\sim 2-4$ au assuming a combined binary mass ranging between $20-100 M_{\odot}$ (Figure 7, top).

Given the nearly edge-on geometry of NaSt1's extended nebula (Mauerhan et al. 2015), we present possible binary origins of variability including (1) eclipses of a circumbinary disk from an optically and geometrically thick circumstellar disk around one of the stars, (2) variations in the line-of-sight optical depth to optical/near-IR-emitting regions due to colliding-wind dust formation, and (3) ellipsoidal variability from a tidally elongated star (Figure 7 , bottom). We favor the colliding-wind dust formation hypothesis; however, future work with $3 \mathrm{D}$ radiative transfer modeling is crucial to reproduce optical and IR light curves that incorporate the line-of-sight optical depth variability throughout the orbit of a highly inclined dust-forming colliding-wind binary. Ultimately, the origin(s) of NaStl's photometric variability is still an open question. Further work and more observations are needed to develop a comprehensive interpretation that satisfies all of the observational constraints including the phase offsets and historical IR variability.

Assuming that NaSt1's periodic photometric variability reflects a 310 day binary orbital period, we addressed whether the binary is undergoing RLOF mass transfer. Based on the CNO-enriched material in NaSt1's nebula and the estimated range of sizes for the Roche-lobe radius of the mass donor star in NaSt1 $\left(\sim 140-450 R_{\odot}\right)$, we suggest that mass transfer could have been triggered by an LBV-like eruptive phase in an Ofpe/ WN9 star in NaSt1 that filled its Roche lobe as it expanded. This interpretation is bolstered with recent observations by Smith et al. (2020) that confirmed Ofpe/WN9 stars can undergo LBV-like eruptions and expand to double the size of their quiescent radius. Owing to the mass loss from its outer envelope, it is possible that the mass donor star in NaSt1 no 
longer resembles an Ofpe/WN9 star. The donor star may have transitioned to either a hotter, earlier WN spectral subtype or a more chemically advanced WC phase. Radio observations of NaSt1 in the GLOSTAR survey (Medina et al. 2019) reveal a spectral index consistent with free-free emission from an ionized stellar wind and reinforces the interpretation that NaSt1 hosts a hot massive star. Given the relation between the observed radio flux and stellar-wind parameters provided by Wright \& Barlow (1975), we estimated a mass-loss rate of $\sim 10^{-4} M_{\odot} \mathrm{yr}^{-1}$, consistent with the upper range of massloss rates exhibited by Galactic WN stars.

Based on the inferred orbital separation and the radius estimates of the star that dominates the ionizing flux and $V$ band emission, we suggest that such a binary system is no longer undergoing mass transfer. NaSt1 still remains an enigmatic system that could provide new insights into an important transitional evolutionary phase of massive stars. Multiwavelength follow-up observations and continued spectroscopic and photometric monitoring will therefore be valuable for addressing the open questions on the nature of NaSt1.

We thank T. Jayasinghe for discussion of the technical details of the filter properties in the ASAS-SN survey. We also thank M. Munoz for an enlightening discussion of Of?p stars. R.M.L. acknowledges the Japan Aerospace Exploration Agency's International Top Young Fellowship (ITYF). A.F.J. M. is grateful for financial assistance from NSERC (Canada). M.M.K. acknowledges the Heising-Simons foundation for support via a Scialog fellowship of the Research Corporation. M.M.K. and A.M.M. acknowledge the Mt. Cuba Astronomical Foundation. A.V.F. is grateful for financial assistance from the TABASGO Foundation, the Christopher R. Redlich Fund, the U.C. Berkeley Miller Institute for Basic Research in Science (in which he is a Miller Senior Fellow), and many individual donors. M.M.K. acknowledges generous support from the David and Lucille Packard Foundation. J.S. is supported by an Australian Government Research Training Program (RTP) Scholarship.

Palomar Gattini-IR (PGIR) is generously funded by Caltech, Australian National University, the Mt. Cuba Astronomical Foundation, the Heising-Simons Foundation, and the Binational Science Foundation. PGIR is a collaborative project among Caltech, Australian National University, University of New South Wales, Columbia University, and the Weizmann Institute of Science. Based in part on observations obtained with the Samuel Oschin 48 inch Telescope at the Palomar Observatory as part of the Zwicky Transient Facility project. ZTF is supported by the National Science Foundation (NSF) under grant AST-1440341 and a collaboration including Caltech, IPAC, the Weizmann Institute for Science, the Oskar Klein Center at Stockholm University, the University of Maryland, the University of Washington, Deutsches Elektronen-Synchrotron and Humboldt University, Los Alamos National Laboratories, the TANGO Consortium of Taiwan, the University of Wisconsin at Milwaukee, and Lawrence Berkeley National Laboratories. Operations are conducted by Caltech Optical Observatories, IPAC, and the University of Washington.

We thank the Las Cumbres Observatory and its staff for its continuing support of the ASAS-SN project. LCOGT observations were performed as part of DDT award 2019B003 to E.G.
ASAS-SN is supported by the Gordon and Betty Moore Foundation through grant GBMF5490 to the Ohio State University, and NSF grants AST-1515927 and AST-1908570. Development of ASAS-SN has been supported by NSF grant AST-0908816, the Mt. Cuba Astronomical Foundation, the Center for Cosmology and AstroParticle Physics at the Ohio State University, the Chinese Academy of Sciences South America Center for Astronomy (CAS- SACA), the Villum Foundation, and George Skestos.

UKIRT is owned by the University of Hawaii (UH) and operated by the UH Institute for Astronomy; operations are enabled through the cooperation of the East Asian Observatory. When the data reported here were acquired, UKIRT was operated by the Joint Astronomy Centre on behalf of the Science and Technology Facilities Council of the UK.

Research at Lick Observatory is partially supported by a generous gift from Google. KAIT and its ongoing operation were made possible by donations from Sun Microsystems, Inc., the Hewlett-Packard Company, AutoScope Corporation, Lick Observatory, the NSF, the University of California, the Sylvia and Jim Katzman Foundation, and the TABASGO Foundation.

This work has made use of data from the Asteroid Terrestrial-impact Last Alert System (ATLAS) project. The Asteroid Terrestrial-impact Last Alert System (ATLAS) project is primarily funded to search for near-Earth objects (NEOs) through NASA grants NN12AR55G, 80NSSC18K0284, and 80NSSC18K1575; byproducts of the NEO search include images and catalogs from the survey area. This work was partially funded by Kepler/K2 grant J1944/80NSSC19K0112 and HST GO-15889, and STFC grants ST/T000198/1 and $\mathrm{ST} / \mathrm{S} 006109 / 1$. The ATLAS science products have been made possible through the contributions of the University of Hawaii Institute for Astronomy, the Queen's University Belfast, the Space Telescope Science Institute, the South African Astronomical Observatory, and The Millennium Institute of Astrophysics (MAS), Chile.

This research made use of Astropy, ${ }^{22}$ a communitydeveloped core Python package for Astronomy (Astropy Collaboration et al. 2013, 2018).

Facilities: PGIR, ATLAS, KAIT, ZTF, ASAS-SN, AEOS (BASS), UKIRT, ESO 1-m.

\section{ORCID iDs}

Samaporn Tinyanont (iD https://orcid.org/0000-00021481-4676

Matthew J. Hankins (iD https://orcid.org/0000-0001-9315-8437

Michael C. B. Ashley (i) https://orcid.org/0000-00031412-2028

Alexei V. Filippenko (iD https://orcid.org/0000-00033460-0103

Mansi M. Kasliwal (D) https://orcid.org/0000-0002-5619-4938

Jon C. Mauerhan (iD https://orcid.org/0000-0002-7555-8741

Anthony F. J. Moffat (ib https://orcid.org/0000-0002-

4333-9755

Nathan Smith (i) https://orcid.org/0000-0001-5510-2424

Roberto Soria (ib https://orcid.org/0000-0002-4622-796X

Peredur M. Williams (i) https://orcid.org/0000-0002-

8092-980X

WeiKang Zheng (i) https://orcid.org/0000-0002-2636-6508

\footnotetext{
22 http://www.astropy.org
} 


\section{References}

Abbott, D. C., Beiging, J. H., Churchwell, E., et al. 1986, ApJ, 303, 239 Astropy Collaboration, Price-Whelan, A. M., Sipőcz, B. M., et al. 2018, AJ, 156,123

Astropy Collaboration, Robitaille, T. P., Tollerud, E., et al. 2013, A\&A, 558, A33

Belczynski, K., Holz, D. E., Bulik, T., et al. 2016, Natur, 534, 512

Bellm, E. C., Kulkarni, S. R., Graham, M. J., et al. 2019, PASP, 131, 018002

Bieging, J. H., Abbott, D. C., \& Churchwell, E. B. 1989, ApJ, 340, 518

Bohannan, B., \& Walborn, N. R. 1989, PASP, 101, 520

Carpenter, J. M. 2001, AJ, 121, 2851

Chambers, K. C., Magnier, E. A., Metcalfe, N., et al. 2016, arXiv:1612.05560

Corcoran, M. F. 2005, AJ, 129, 2018

Crowther, P. A. 2007, ARA\&A, 45, 177

Crowther, P. A., \& Smith, L. J. 1999, MNRAS, 308, 82

Cutri, R. M., Skrutskie, M. F., van Dyk, S., et al. 2003, yCat, II/246

Damineli, A. 1996, ApJL, 460, L49

Damineli, A., Hillier, D. J., Corcoran, M. F., et al. 2008, MNRAS, 384, 1649

De, K., Ashley, M. C. B., Andreoni, I., et al. 2020b, ApJL, 901, L7

De, K., Hankins, M. J., Kasliwal, M. M., et al. 2020a, PASP, 132, 025001

De Marco, O., \& Izzard, R. G. 2017, PASA, 34, e001

Djurašević, G., Vince, I., \& Atanacković, O. 2008, AJ, 136, 767

Eggleton, P. P. 1983, ApJ, 268, 368

Eldridge, J. J., Stanway, E. R., Xiao, L., et al. 2017, PASA, 34, e058

Fabrika, S. 2004, Astrophys. Space Phys. Res., 12, 1

Filippenko, A. V., Li, W. D., Treffers, R. R., et al. 2001, in IAU Coll. 183: Small Telescope Astronomy on Global Scales, 121

Gaia Collaboration 2018, yCat, I/345

Ganeshalingam, M., Li, W., Filippenko, A. V., et al. 2010, ApJS, 190, 418

Gehrz, R. D., Hayward, T. L., Houck, J. R., et al. 1995, ApJ, 439, 417

Götberg, Y., de Mink, S. E., Groh, J. H., et al. 2018, A\&A, 615, A78

Götberg, Y., de Mink, S. E., McQuinn, M., et al. 2020, A\&A, 634, A134

Groh, J. H., Meynet, G., \& Ekström, S. 2013, A\&A, 550, L7

Grundstrom, E. D., Gies, D. R., Hillwig, T. C., et al. 2007, ApJ, 667, 505

Grunhut, J. H., Wade, G. A., Neiner, C., et al. 2017, MNRAS, 465, 2432

Hackwell, J. A., Warren, D. W., Chatelain, M. A., et al. 1990, Proc. SPIE, 1235,171

Hamann, W.-R., Gräfener, G., Liermann, A., et al. 2019, A\&A, 625, A57

Hawarden, T. G., Leggett, S. K., Letawsky, M. B., et al. 2001, MNRAS, 325,563

Heger, A., Fryer, C. L., Woosley, S. E., et al. 2003, ApJ, 591, 288

Heinze, A. N., Tonry, J. L., Denneau, L., et al. 2018, AJ, 156, 241

Hendrix, T., Keppens, R., van Marle, A. J., et al. 2016, MNRAS, 460, 3975

Howarth, I. D., Walborn, N. R., Lennon, D. J., et al. 2007, MNRAS, 381, 433

Jayasinghe, T., Stanek, K. Z., Kochanek, C. S., et al. 2020, MNRAS, 493, 4186 Klagyivik, P., \& Szabados, L. 2009, A\&A, 504, 959

Kochanek, C. S., Shappee, B. J., Stanek, K. Z., et al. 2017, PASP, 129, 104502 Landolt, A. U. 1992, AJ, 104, 340

Lau, R. M., Eldridge, J. J., Hankins, M. J., et al. 2020, ApJ, 898, 74

Leggett, S. K., Hawarden, T. G., Currie, M. J., et al. 2003, MNRAS, 345, 144

Leitherer, C., Chapman, J. M., \& Koribalski, B. 1997, ApJ, 481, 898

Li, W., Filippenko, A. V., Chornock, R., et al. 2003, PASP, 115, 844

Mahy, L., Hutsemékers, D., Nazé, Y., et al. 2017, A\&A, 599, A61

Martins, F., Genzel, R., Hillier, D. J., et al. 2007, A\&A, 468, 233

Masci, F. J., Laher, R. R., Rusholme, B., et al. 2019, PASP, 131, 018003

Massey, P., \& Conti, P. S. 1983, PASP, 95, 440

Mauerhan, J., Smith, N., Van Dyk, S. D., et al. 2015, MNRAS, 450, 2551
Medina, S.-N. X., Urquhart, J. S., Dzib, S. A., et al. 2019, A\&A, 627, A175

Meynet, G., \& Maeder, A. 2005, A\&A, 429, 581

Moe, M., \& Di Stefano, R. 2017, ApJS, 230, 15

Moffat, A. F. J., Niemela, V. S., \& Marraco, H. G. 1990, ApJ, 348, 232

Monnier, J. D., Tuthill, P. G., \& Danchi, W. C. 1999, ApJL, 525, L97

Moore, A. M., \& Kasliwal, M. M. 2019, NatAs, 3, 109

Morris, S. L., \& Naftilan, S. A. 1993, ApJ, 419, 344

Munoz, M. S., Wade, G. A., Nazé, Y., et al. 2020, MNRAS, 492, 1199

Nassau, J. J., \& Stephenson, C. B. 1963, LS, C04, 0

Nazé, Y., Walborn, N. R., Morrell, N., et al. 2015, A\&A, 577, A107

Paczyński, B. 1967, AcA, 17, 355

Panagia, N., \& Felli, M. 1975, A\&A, 39, 1

Podsiadlowski, P., Joss, P. C., \& Hsu, J. J. L. 1992, ApJ, 391, 246

Rajagopal, J., Menut, J.-L., Wallace, D., et al. 2007, ApJ, 671, 2017

Rate, G., \& Crowther, P. A. 2020, MNRAS, 493, 1512

Rodrigo, C., \& Solano, E. 2020, in Contributions to the XIV.0 Scientific Meeting (virtual) of the Spanish Astronomical Society, 182

Rodrigo, C., Solano, E., \& Bayo, A. 2012, IVOA Working Draft 15 October 2012

Rosslowe, C. K., \& Crowther, P. A. 2015, MNRAS, 447, 2322

Sana, H., de Mink, S. E., de Koter, A., et al. 2012, Sci, 337, 444

Sander, A. A. C., \& Vink, J. S. 2020, MNRAS, 499, 873

Shappee, B. J., Prieto, J. L., Grupe, D., et al. 2014, ApJ, 788, 48

Smith, K. W., Smartt, S. J., Young, D. R., et al. 2020, PASP, 132, 085002

Smith, N. 2010, MNRAS, 402, 145

Smith, N. 2014, ARA\&A, 52, 487

Smith, N., Andrews, E., Moe, M., et al. 2020, MNRAS, 492, 5897

Smith, N., Andrews, J. E., Rest, A., et al. 2018, MNRAS, 480, 1466

Smith, N., Gehrz, R. D., Campbell, R., et al. 2011b, MNRAS, 418, 1959

Smith, N., Gehrz, R. D., Humphreys, R. M., et al. 1999, AJ, 118, 960

Smith, N., Gehrz, R. D., Stahl, O., et al. 2002, ApJ, 578, 464

Smith, N., Li, W., Filippenko, A. V., et al. 2011a, MNRAS, 412, 1522

Smith, N., \& Morse, J. A. 2004, ApJ, 605, 854

Soraisam, M. D., Bildsten, L., Drout, M. R., et al. 2020, ApJ, 893, 11

Stahl, B. E., Zheng, W., de Jaeger, T., et al. 2019, MNRAS, 490, 3882

Stetson, P. B. 1987, PASP, 99, 191

Su, K. Y. L., Rieke, G. H., Malhotra, R., et al. 2013, ApJ, 763, 118

Sukhbold, T., Ertl, T., Woosley, S. E., et al. 2016, ApJ, 821, 38

Tonry, J. L., Denneau, L., Heinze, A. N., et al. 2018, PASP, 130, 064505

Tonry, J. L., Stubbs, C. W., Lykke, K. R., et al. 2012, ApJ, 750, 99

Tuthill, P. G., Monnier, J. D., \& Danchi, W. C. 1999, Natur, 398, 487

Tuthill, P. G., Monnier, J. D., Lawrance, N., et al. 2008, ApJ, 675, 698

Usov, V. V. 1991, MNRAS, 252, 49

van der Bliek, N. S., Manfroid, J., \& Bouchet, P. 1996, A\&AS, 119, 547

van der Hucht, K. A., Williams, P. M., Morris, P. W., et al. 1997, Luminous Blue Variables: Massive Stars in Transition, 120, 211

van der Hucht, K. A., Williams, P. M., van Genderen, A. M., et al. 1989, in IAU Coll. 113: Physics of Luminous Blue Variables, 301

Vanbeveren, D., De Donder, E., Van Bever, J., et al. 1998, NewA, 3, 443

Walborn, N. R. 1973, AJ, 78, 1067

Walborn, N. R. 1977, ApJ, 215, 53

Williams, P. M. 2019, MNRAS, 488, 1282

Williams, P. M., Chu, Y.-H., Gruendl, R. A., et al. 2013, MNRAS, 431, 1160

Williams, P. M., Cohen, M., van der Hucht, K. A., et al. 1995, MNRAS, 275,889

Williams, P. M., van der Hucht, K. A., \& Thé, P. S. 1987, A\&A, 182, 91

Wright, A. E., \& Barlow, M. J. 1975, MNRAS, 170, 41 